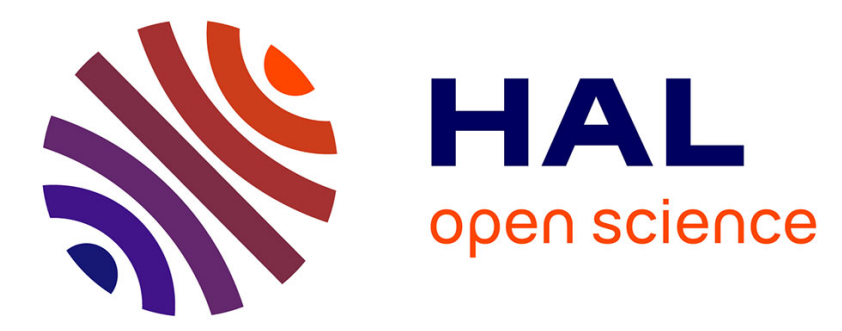

\title{
Saint-Venant's problem for a second-order piezoelectric prismatic bar
}

\author{
Stefano Vidoli, Romesh C. Batra, Francesco Dell'Isola
}

\section{To cite this version:}

Stefano Vidoli, Romesh C. Batra, Francesco Dell'Isola. Saint-Venant's problem for a second-order piezoelectric prismatic bar. International Journal of Engineering Science, 2000, pp.25. hal-00501519

\section{HAL Id: hal-00501519 https://hal.science/hal-00501519}

Submitted on 12 Jul 2010

HAL is a multi-disciplinary open access archive for the deposit and dissemination of scientific research documents, whether they are published or not. The documents may come from teaching and research institutions in France or abroad, or from public or private research centers.
L'archive ouverte pluridisciplinaire HAL, est destinée au dépôt et à la diffusion de documents scientifiques de niveau recherche, publiés ou non, émanant des établissements d'enseignement et de recherche français ou étrangers, des laboratoires publics ou privés. 


\title{
Saint-Venant's problem for a second-order piezoelectric prismatic bar
}

\author{
S. Vidoli ${ }^{a}$, R.C. Batra ${ }^{b, *}$, F. dell'Isola ${ }^{a}$ \\ ${ }^{a}$ Dipartimento di Ingegneria Strutturale e Geotecnica, Universitá di Roma 'La Sapienza', 00184 Roma, Italy \\ ${ }^{\mathrm{b}}$ Department of Engineering Science and Mechanics, Virginia Polytechnic Institute and State University, MC 0219, \\ Blacksburg, VA 24061, USA
}

Received 27 October 1998; received in revised form 6 January 1999; accepted 6 January 1999

\begin{abstract}
For a homogeneous, transversely isotropic, second-order piezoelectric and infinitesimally prebent, prepolarized and prestretched prismatic bar, we find second-order Saint-Venant solutions. It is found that the electric field alone does not introduce any bending in the second-order piezoelectric bar. However, the electric field induced by the bending of the bar can be used to ascertain the magnitude and direction of the bending vector. The curvature of the centroidal axis of the bar can be changed by the simultaneous application of the electric field and the bending moment. (c) 1999 Elsevier Science Ltd. All rights reserved.
\end{abstract}

Keywords: Signorini's expansion; Saint-Venant's solutions; Semi-inverse method

\section{Introduction}

Saint-Venant [1,2] studied the extension, bending, torsion and flexure of a homogeneous prismatic body made of an isotropic linear elastic material. Iesan [3-6] has analysed the SaintVenant problem for inhomogeneous and anisotropic linear elastic bodies, elastic dielectrics and microstretch elastic solids. Dell'Isola and Rosa [7,8] and Dávi [9] have investigated the problem for linear piezoelectric materials and dell'Isola and Batra [10] for linear elastic isotropic porous solids. Batra and Yang [11] have proved Toupin's version [12] of the Saint-Venant principle for a linear piezoelectric bar.

Rivlin [13] has used the second-order elasticity theory to explain the Poynting effect [14], i.e., the axial elongation of an elastic bar subjected to torques only at the end faces is proportional to the

\footnotetext{
* Corresponding author. Tel.: +001-540-231-6051; fax: +001-540-231-4574.

E-mail address: rbatra@vt.edu (R.C. Batra)
} 
square of the angular twist. Rivlin showed that the elongation was proportional to the secondorder elasticities. Signorini [15] proposed a perturbation method to reduce the solution of a nonlinear elastic problem to that of a series of nonhomogeneous linear elastic traction boundary value problems in which the loads for the $n$th problem depend upon the solution of the previous $(n-1)$ problems. These loads must satisfy compatibility conditions for the $n$ th-order problem to have a solution. Truesdell and Noll [16] have reviewed the pertinent literature on the Poynting effect and the Signorini method. Green and Adkins [17] pointed out that when the displacements and infinitesimal rotations of the centroid of one end face vanish, then the compatibility conditions for the loads in the $n$th order Signorini problem are automatically satisfied. Green and Adkins [17] and Green and Shield [18] have studied the Poynting effect in nonlinear elastic prismatic bodies.

Recently, dell'Isola et al. [19,20] used the Signorini expansion method to find a solution of the Saint-Venant problem for a prismatic bar made of a second-order elastic material with the first-order solution corresponding to either an infinitesimal twist or small bending and stretching. Batra et al. [21] used the Signorini expansion method to analyze electromechanical deformations of a transversely isotropic circular cylindrical bar made of a second-order piezoelectric material for which constitutive relations had been derived by Yang and Batra [22]. The first-order deformations were assumed to be an infinitesimal twist and small electric field. Here Batra et al.'s [21] work is extended to a prismatic body of a general cross-section which is initially twisted, stretched and electrically polarized by a small amount. For a bar subjected to bending moments only at the end faces, it is found that there is a second-order Poisson's effect proportional to the square of the magnitude of the bending vector and a torsional effect proportional to the square of the axial distance from the 'clamped' end. Other second-order effects are stated in the paper.

\section{Formulation of the problem}

We consider a transversely isotropic and homogeneous prismatic body of cross-section $\mathscr{A}$ and length $\ell$ occupying the domain $\Omega=\mathscr{A} \times[0, \ell]$ in the stress and polarization free configuration with its axis along the unit vector $\mathbf{e}$ that is also the direction of transverse isotropy. In the referential description of deformation, the balance of linear momentum, the balance of moment of momentum, the Maxwell law for the electric displacement with the free body charge density set equal to zero and the boundary conditions are

$$
\begin{aligned}
& \operatorname{Div}\left(\mathbf{T}+\mathbf{T}^{E}\right)=\mathbf{0} \text { in } \Omega, \\
& \left(\mathbf{T}+\mathbf{T}^{E}\right) \mathbf{F}^{T}=\mathbf{F}\left(\mathbf{T}+\mathbf{T}^{E}\right)^{T} \text { in } \Omega, \\
& \operatorname{Div} \mathbb{D}=0 \text { in } \Omega, \\
& \left(\mathbf{T}+\mathbf{T}^{E}\right) \mathbf{N}=\mathbf{0}, \mathbb{D} \cdot \mathbf{N}=0 \text { on } \partial \mathscr{A} \times[0, \ell], \\
& \left(\mathbf{T}+\mathbf{T}^{E}\right) \mathbf{e}=\mathbf{f}, \mathbb{D} \cdot \mathbf{e}=q \text { on } \mathscr{A}_{0} \text { and } \mathscr{A}_{\ell}, \\
& \mathbf{u}=\mathbf{0}, \mathbf{H}-\mathbf{H}^{T}=\mathbf{0}, \text { and } \psi=0 \text { at point } C .
\end{aligned}
$$


Here $\mathbf{T}$ is the first Piola-Kirchhoff stress tensor, $\mathbf{T}^{E}$ the first Piola-Kirchhoff-Maxwell stress tensor, $\mathbb{D}$ the referential electric displacement, Div the divergence operator in the reference configuration, $\mathbf{F}$ the deformation gradient, $\mathbf{N}$ a unit outward normal to the mantle in the reference configuration, $\mathbf{f}$ the surface traction per unit undeformed area, $q$ the charge density, $\mathbf{u}=\mathbf{x}-\mathbf{X}$ the displacement of a material point that occupied place $\mathbf{X}$ in the reference configuration and is at place $\mathbf{x}$ in the present configuration, $\mathbf{H}=\mathrm{Grad} \mathbf{u}$ the displacement gradient, Grad the gradient operator with respect to coordinates in the reference configuration, $\psi$ the electric potential and point $C$ is the centroid of the cross-section $\mathscr{A}_{0} \equiv \mathscr{A} \times\{0\}$. The clamping condition (6) eliminates trivial solutions of the problem.

For the problem to have a solution, $\mathbf{f}$ and $q$ must satisfy

$$
\begin{aligned}
& \int_{\mathscr{A}_{0}} \mathbf{f} \mathrm{d} A+\int_{\mathscr{A}_{\ell}} \mathbf{f} \mathrm{d} A=\mathbf{0}, \int_{\mathscr{A}_{0}} q \mathrm{~d} A+\int_{\mathscr{A}_{\ell}} q \mathrm{~d} A=0, \\
& \int_{\mathscr{A}_{0}} \mathbf{x} \wedge \mathbf{f} \mathrm{d} A+\int_{\mathscr{A}_{\ell}} \mathbf{x} \wedge \mathbf{f} \mathrm{d} A=\mathbf{0},
\end{aligned}
$$

where

$$
\begin{aligned}
& \mathbf{a} \wedge \mathbf{b}=\mathbf{a} \otimes \mathbf{b}-\mathbf{b} \otimes \mathbf{a}, \\
& (\mathbf{a} \otimes \mathbf{b}) \mathbf{c}=(\mathbf{b} \cdot \mathbf{c}) \mathbf{a},
\end{aligned}
$$

for arbitrary vectors a, b and c. Eqs. (4), (5) and (7) imply that

$$
\begin{aligned}
& \left(\int_{\mathscr{A}}\left(\mathbf{T}+\mathbf{T}^{E}\right) \mathbf{e} \mathrm{d} A\right)^{\prime}=\mathbf{0}, \quad\left(\int_{\mathscr{A}} \mathbb{D} \cdot \mathbf{e} \mathrm{d} A\right)^{\prime}=0, \\
& \left(\int_{\mathscr{A}} \mathbf{x} \wedge\left(\mathbf{T}+\mathbf{T}^{E}\right) \mathbf{e} \mathrm{d} A\right)^{\prime}+\left.\mathbf{x}\right|_{\mathbf{r}=\mathbf{0}} ^{\prime} \wedge \int_{\mathscr{A}}\left(\mathbf{T}+\mathbf{T}^{E}\right) \mathbf{e} \mathrm{d} A=\mathbf{0},
\end{aligned}
$$

where we have taken the origin of our coordinate system at the centroid of the cross-section $\mathscr{A}_{0}$ and set

$$
\mathbf{X}=\mathbf{r}+z \mathbf{e} .
$$

In Eq. (9) a prime indicates differentiation with respect to the axial coordinate, $z$. Recall that

$$
\mathbf{T}=J \boldsymbol{\sigma} \mathbf{F}^{-1^{T}}, \quad \mathbf{T}^{E}=J \boldsymbol{\sigma}^{E} \mathbf{F}^{-1^{T}}, \quad \mathbb{D}=J \mathbf{F}^{-1} \mathbf{D},
$$

where $J=\operatorname{det} \mathbf{F}, \boldsymbol{\sigma}$ is the Cauchy stress tensor, $\boldsymbol{\sigma}^{E}$ the Cauchy-Maxwell stress tensor and $\mathbf{D}$ the electric displacement in the present configuration.

For a piezoelectric material, we introduce, in the present configuration, electric field $\hat{\mathbf{E}}$ and electric polarization $\mathbf{P}$ through

$$
\mathbf{D}=\mathbf{P}+\hat{\mathbf{E}} .
$$

Quantities $\mathbf{P}$ and $\hat{\mathbf{E}}$ are related to their counterparts $\boldsymbol{\Pi}$ and $\mathbf{W}$ in the reference configuration by

$$
\boldsymbol{\Pi}=J \mathbf{F}^{-1} \mathbf{P}, \mathbf{W}=\mathbf{F}^{T} \hat{\mathbf{E}}=-\operatorname{Grad} \psi .
$$


The existence of $\psi$ is guaranteed by

$$
\oint \hat{\mathbf{E}} \cdot \mathrm{d} \mathbf{x}=0
$$

where the integration is on any closed curve in $\Omega$.

In order to complete the formulation of the problem we need to give the constitutive relations. We choose $\boldsymbol{\sigma}$ and $\boldsymbol{\sigma}^{E}$ to be symmetric tensors so that the balance of moment of momentum (2) is identically satisfied. Following Abraham et al. (see [23], Eqn.3.6,22,23) we take

$$
\boldsymbol{\sigma}^{E}=(\mathbf{P} \otimes \hat{\mathbf{E}})_{s}+\hat{\mathbf{E}} \otimes \hat{\mathbf{E}}-\frac{1}{2} \hat{E}^{2} \mathbf{1}
$$

where

$$
(\mathbf{a} \otimes \mathbf{b})_{s}=(\mathbf{a} \otimes \mathbf{b}+\mathbf{b} \otimes \mathbf{a}) / 2,
$$

$\hat{E}$ is the magnitude of $\hat{\mathbf{E}}$ and $\mathbf{1}$ is the identity tensor. We assume that the prismatic body is made of a transversely isotropic second-order piezoelectric material. For such a material, Yang and Batra [22] have derived constitutive relations for the second Piola-Kirchhoff stress tensor $\mathbf{S}$ and the referential polarization $\boldsymbol{\Pi}$. We use those to obtain expressions for $\mathbf{T}$ and $\mathbb{D}$ and retain terms upto second-order in $\mathbf{H}$ and $\mathbf{W}$. These rather long expressions are omitted. However, the expressions used herein to obtain a solution of the problem by the method of Signorini's series expansion are given in Appendix A.

\section{Signorini's expansion}

We write the displacement field $\mathbf{u}$ and the electric field $\mathbf{W}$ as

$$
\mathbf{u}=w \mathbf{e}+\mathbf{v}, \quad \mathbf{W}=-\left(\psi^{\prime} \mathbf{e}+\operatorname{grad} \psi\right) .
$$

That is, $w$ and $\mathbf{v}$ are the axial and in-plane components of the displacement $\mathbf{u}$ of a point and $\psi^{\prime}$ and $\operatorname{grad} \psi$ equal the axial and in-plane components of $\mathbf{W}$. The operators grad and div signify, respectively, the two-dimensional gradient and divergence operators with respect to referential coordinates in the cross-section $\mathscr{A}$.

The displacement $\mathbf{u}$, the electric potential $\psi$, surface tractions $\mathbf{f}$ and surface charge $q$ are assumed to have a series expansion

$$
\begin{aligned}
& \mathbf{u}=\eta \dot{\mathbf{u}}+\eta^{2} \ddot{\mathbf{u}}+\cdots, \quad \psi=\eta \dot{\psi}+\eta^{2} \ddot{\psi}+\cdots, \\
& \mathbf{f}=\eta \dot{\mathbf{f}}+\eta^{2} \ddot{\mathbf{f}}+\cdots, \quad q=\eta \dot{q}+\eta^{2} \ddot{q}+\cdots,
\end{aligned}
$$

where $\eta$ is a small, yet to be identified, parameter in the problem. Substitutions for $\mathbf{u}$ and $\psi$ in the expressions for the constitutive relations for $\mathbf{T}$ and $\mathbb{D}$ give

$$
\mathbf{T}=\eta \dot{\mathbf{T}}+\eta^{2} \ddot{\mathbf{T}}+\cdots, \quad \mathbb{D}=\eta \ddot{\mathbb{D}}+\eta^{2} \ddot{\mathbb{D}}+\cdots, \quad \mathbf{T}^{E}=\eta^{2} \ddot{\mathbf{T}}^{E}+\cdots
$$


Expressions for $\dot{\mathbf{T}}, \ddot{\mathbf{T}}, \ddot{\mathbf{T}}^{E}, \ddot{\mathbb{D}}$ and $\ddot{\mathbb{D}}$ are given in the Appendix. Substituting from Eqs. (18) and (19) into the balance laws (1)-(3), the boundary conditions (4)-(6) and integrability conditions (9) and equating like powers of $\eta$ on both sides of these equations, we arrive at the following equations for the first and second-order problems:

$\operatorname{Div} \dot{\mathbf{T}}=\mathbf{0}, \quad \operatorname{Div} \dot{\mathbb{D}}=0$ in $\Omega$,

$$
\begin{aligned}
& \dot{\mathbf{T}} \mathbf{N}=\mathbf{0}, \quad \dot{\mathbb{D}} \cdot \mathbf{N}=0 \text { on } \partial \mathscr{A} \times[0, \ell], \\
& \dot{\mathbf{T}} \mathbf{e}=\dot{\mathbf{f}}, \quad \dot{\mathbb{D}} \cdot \mathbf{e}=\dot{q} \text { on } \mathscr{A}_{0} \text { and } \mathscr{A}_{\ell}, \\
& \left(\int_{\mathscr{A}} \dot{\mathbf{T}} \mathrm{d} A\right)^{\prime}=\mathbf{0}, \quad\left(\int_{\mathscr{A}} \dot{\mathbb{D}} \cdot \mathbf{e} \mathrm{d} A\right)^{\prime}=0, \\
& \left(\int_{\mathscr{A}} \mathbf{X} \wedge \dot{\mathbf{T}} \mathbf{e} A\right)^{\prime}+\left.\mathbf{X}\right|_{\mathbf{r}=\mathbf{0}} ^{\prime} \wedge \int_{\mathscr{A}} \dot{\mathbf{T}} \mathrm{d} A=\mathbf{0} ;
\end{aligned}
$$

$$
\begin{aligned}
& \operatorname{Div}\left(\ddot{\mathbf{T}}+\ddot{\mathbf{T}}^{E}\right)=\mathbf{0}, \quad \operatorname{Div}\left(\ddot{\mathbb{D}}+j \dot{\mathbf{W}}-2(\dot{\mathbf{H}})_{s} \dot{\mathbf{W}}\right)=0 \text { in } \Omega, \\
& \left(\ddot{\mathbf{T}}+\ddot{\mathbf{T}}^{E}\right) \mathbf{N}=\mathbf{0}, \quad\left(\ddot{\mathbb{D}}+j \dot{\mathbf{W}}-2(\dot{\mathbf{H}})_{s} \dot{\mathbf{W}}\right) \cdot \mathbf{N}=0 \text { on } \partial \mathscr{A} \times[0, \ell], \\
& \left(\ddot{\mathbf{T}}+\ddot{\mathbf{T}}^{E}\right) \mathbf{e}=\ddot{\mathbf{f}}, \quad\left(\ddot{\mathbb{D}}+j \dot{\mathbf{W}}-2(\dot{\mathbf{H}})_{s} \dot{\mathbf{W}}\right) \cdot \mathbf{e}=\ddot{q} \text { on } \mathscr{A}_{0} \text { and } \mathscr{A} \ell, \\
& \left(\int_{\mathscr{A}}\left(\ddot{\mathbf{T}}+\ddot{\mathbf{T}}^{E}\right) \mathbf{e} \mathrm{d} A\right)^{\prime}=\mathbf{0}, \quad\left(\int_{\mathscr{A}}\left(\ddot{\mathbb{D}}+j \dot{\mathbf{W}}-2(\dot{\mathbf{H}})_{s} \dot{\mathbf{W}}\right) \cdot \mathbf{e} \mathrm{d} A\right)^{\prime}=0, \\
& \left(\int_{\mathscr{A}}\left[\mathbf{X} \wedge\left(\ddot{\mathbf{T}}+\ddot{\mathbf{T}}^{E}\right) \mathbf{e}+\dot{\mathbf{u}} \wedge \dot{\mathbf{T}} \mathbf{e}\right] \mathrm{d} A\right)^{\prime}+\mathbf{e} \wedge \int_{\mathscr{A}}\left(\ddot{\mathbf{T}}+\ddot{\mathbf{T}}^{E}\right) \mathbf{e} \mathrm{d} A+\dot{\mathbf{u}}_{\mathbf{r}=\mathbf{0}}^{\prime} \wedge \int_{\mathscr{A}} \dot{\mathbf{T}} \mathbf{e} \mathrm{d} A=\mathbf{0} .
\end{aligned}
$$

We decompose $\ddot{\mathbf{T}}$ and $\ddot{\mathbb{D}}$ as

$$
\ddot{\mathbf{T}}=\ddot{\overline{\mathbf{T}}}+\ddot{\mathbf{T}}^{s}, \quad \ddot{\mathbb{D}}=\ddot{\overline{\mathbb{D}}}+\ddot{\mathbb{D}}^{s},
$$

where $\ddot{\overline{\mathbf{T}}}$ and $\ddot{\overline{\mathbb{D}}}$ are related to $\ddot{\mathbf{u}}$ and $\ddot{\psi}$ in the same way as $\dot{\mathbf{T}}$ and $\dot{\mathbb{D}}$ to $\dot{\mathbf{u}}$ and $\dot{\psi}$. Expressions for $\ddot{\overline{\mathbf{T}}}$ and $\ddot{\overline{\mathbb{D}}}$ in terms of $\ddot{\mathbf{u}}$ and $\ddot{\psi}$ are

$$
\begin{aligned}
2 \ddot{\overline{\mathbf{T}}}= & \tilde{\mu}(\operatorname{grad} \ddot{\mathbf{v}})_{s}+\left[\left(c_{3}+\lambda\right) \ddot{w}^{\prime}+\lambda \operatorname{div} \ddot{\mathbf{v}}-e_{2} \ddot{\psi}^{\prime}\right] \hat{\mathbf{I}} \\
& +\left\{\left[\tilde{\mu}\left(\ddot{\mathbf{v}}^{\prime}+\operatorname{grad} \ddot{w}\right)-e_{3} \operatorname{grad} \ddot{\psi}\right] \otimes \mathbf{e}\right\}_{s} \\
& +\left[2\left(c_{1}+\lambda / 2+c_{3}+c_{4}+\mu\right) \ddot{w}^{\prime}+\left(c_{3}+\lambda\right) \operatorname{div} \ddot{\mathbf{v}}-\left(e_{1}+e_{2}+2 e_{3}\right) \ddot{\psi}^{\prime}\right] \mathbf{e} \otimes \mathbf{e}, \\
\ddot{\overline{\mathbb{D}}}= & \left(2 \epsilon_{2}-1\right) \operatorname{grad} \ddot{\psi}-e_{3}\left(\ddot{\mathbf{v}}^{\prime}+\operatorname{grad} \ddot{w}\right) \\
& +\left[2\left(\epsilon_{1}+\epsilon_{2}-1\right) \ddot{\psi}^{\prime}-\left(e_{1}+e_{2}+2 e_{3}\right) \ddot{w}^{\prime}-e_{2} \operatorname{div} \ddot{\mathbf{v}}\right] \mathbf{e} .
\end{aligned}
$$

Here $\hat{\mathbf{I}}$ is the two-dimensional identity tensor and $c_{1}, c_{3}, c_{4}, e_{1}, e_{2}, e_{3}, \epsilon_{1}$ and $\epsilon_{2}$ are material parameters and $\tilde{\mu}=\left(c_{4}+2 \mu\right) / 2$ is the shear modulus in the direction of transverse isotropy. Eq. (23) with two superimposed dots replaced by a superimposed dot are constitutive relations for a linear transversely isotropic piezoelectric material. We assume that $\lambda, \mu, c_{1}, c_{3}$, 
$c_{4}, e_{1}, e_{2}, e_{3}, \epsilon_{1}$ and $\epsilon_{2}$ are such that the strain energy density is positive definite so that the solution of a traction boundary value problem for a linear piezoelectric body is unique to within a trivial solution. Substituting from Eq. (12) into Eq. (21) and rewriting equations in the same form as Eq. (20), we obtain

$\operatorname{Div} \ddot{\overline{\mathbf{T}}}=\mathbf{b}^{s}, \quad \operatorname{Div} \ddot{\overline{\mathbb{D}}}=c^{s}$ in $\Omega$,

$$
\begin{aligned}
& \ddot{\overline{\mathbf{T}} \mathbf{N}}=\mathbf{f}^{m s}, \ddot{\overline{\mathbb{D}}} \cdot \mathbf{N}=q^{m s} \text { on } \partial \mathscr{A} \times[0, \ell], \\
& \int_{\mathscr{A}} \ddot{\overline{\mathbf{T}}} \mathbf{e} \mathrm{d}=\int_{\mathscr{A}} \ddot{\mathbf{f}} \mathrm{d} A+\mathbf{R}^{f s}, \quad \int_{\mathscr{A}} \ddot{\overline{\mathbb{D}}} \cdot \mathbf{e} \mathrm{d} A=\int_{\mathscr{A}} \ddot{q} \mathrm{~d} A+R^{q s}, \\
& \int_{\mathscr{A}} \mathbf{X} \wedge(\ddot{\overline{\mathbf{T}}} \mathbf{e}) \mathrm{d} A=\int_{\mathscr{A}} \mathbf{X} \wedge \ddot{\mathbf{f}} \mathrm{d} A+\mathbf{R}^{m s}, \\
& \int_{\mathscr{A}} \ddot{\overline{\mathbf{T}}}^{\prime} \mathbf{e} d A=\mathbf{h}^{s}, \quad \int_{\mathscr{A}} \ddot{\overline{\mathbb{D}}}^{\prime} \cdot \mathbf{e} \mathrm{d} A=i^{s}, \\
& \int_{\mathscr{A}}(\mathbf{X} \wedge \ddot{\overline{\mathbf{T}}} \mathbf{e})^{\prime} \mathrm{d} A+\mathbf{e} \wedge \int_{\mathscr{A}} \ddot{\overline{\mathbf{T}}} \mathbf{e} \mathrm{d} A=\mathbf{g}^{s},
\end{aligned}
$$

where

$$
\begin{aligned}
& \mathbf{b}^{s}=-\operatorname{Div} \ddot{\mathbf{T}}^{s e}, \quad c^{s}=-\operatorname{Div} \ddot{\mathbb{D}}^{s p}, \quad \mathbf{f}^{m s}=-\ddot{\mathbf{T}}^{s e} \mathbf{N}, \quad q^{m s}=-\ddot{\mathbb{D}}^{s p} \cdot \mathbf{N}, \\
& \mathbf{R}^{f s}=-\int_{\mathscr{A}} \ddot{\mathbf{T}}^{s e} \mathbf{e} \mathrm{d} A, \quad \mathbf{R}^{m s}=-\int_{\mathscr{A}} \mathbf{X} \wedge\left(\ddot{\mathbf{T}}^{s e} \mathbf{e}\right) \mathrm{d} A, \quad R^{q s}=-\int_{\mathscr{A}} \ddot{\mathbb{D}}^{s p} \cdot \mathbf{e} \mathrm{d} A, \\
& \mathbf{h}^{s}=-\int_{\mathscr{T}}\left(\ddot{\mathbf{T}}^{s e}\right)^{\prime} \mathbf{e} \mathrm{d} A, \\
& \mathbf{g}^{s}=-\int_{\mathscr{A}}\left(\mathbf{X} \wedge \ddot{\mathbf{T}}^{s e} \mathbf{e}+\dot{\mathbf{u}} \wedge \dot{\mathbf{T}} \mathbf{e}\right)^{\prime} \mathrm{d} A-\mathbf{e} \wedge \int_{\mathscr{A}} \ddot{\mathbf{T}}^{s e} \mathbf{e} \mathrm{d} A-\left.\dot{\mathbf{u}}\right|_{\mathbf{r}=\mathbf{0}} ^{\prime} \wedge \int_{\mathscr{A}} \dot{\mathbf{T}} \mathbf{e} \mathrm{d} A, \\
& i^{s}=-\int_{\mathscr{A}}\left(\ddot{\mathbb{D}}^{s p}\right)^{\prime} \cdot \mathbf{e} \mathrm{d} A, \\
& \ddot{\mathbf{T}}^{s e}=\ddot{\mathbf{T}}^{s}+\ddot{\mathbf{T}}^{E}, \quad \ddot{\mathbb{D}}^{s p}=\ddot{\mathbb{D}}^{s}+\dot{J} \dot{\mathbf{W}}-2(\dot{\mathbf{H}})_{s} \dot{\mathbf{W} .}
\end{aligned}
$$

We assume that the first-order deformation corresponds to the infinitesimal bending, extension and polarization of the bar. That is,

$$
\begin{aligned}
& \dot{\psi}=z(\omega+b \epsilon), \\
& \dot{\mathbf{u}}=\tilde{v}(\mathbf{r} \otimes * \mathbf{r})_{s} \mathbb{b}-\frac{z^{2}}{2}(* \mathbb{b})-v(a \omega+\epsilon) \mathbf{r}+z(a \omega+\epsilon+* \mathbb{b} \cdot \mathbf{r}) \mathbf{e},
\end{aligned}
$$

where

$$
a=e_{2} / c_{3}, \quad v=\lambda / 2(\lambda+\mu), \quad * \mathbf{r}=\mathbf{e} \times \mathbf{r}, \quad b=c_{3} / e_{2}, \quad \tilde{v}=\left(c_{3}+\lambda\right) / 2(\lambda+\mu),
$$


$\epsilon, \omega$ and to characterize the infinitesimal extension, polarization and bending of the prismatic body. Note that vector bo is in the cross-section $\mathscr{A}$. Relations between $\omega, \epsilon$ and $b$ in terms of loads and electric charge applied at the end faces are given below as Eqs. (46) and (47). In order for the deformations caused by the bending, extension and the electric field to be of the same order of magnitude, $a \omega=\epsilon=|\mathfrak{b}| R$ where $2 R$ is the diameter of the cross-section, i.e., the diameter of the smallest circle enclosing the cross-section $\mathscr{A}$ and $|\mathfrak{b}|$ is the magnitude of $b$. The small parameter $\eta$ in Eq. (18) can be identified with $a \omega, \epsilon$ or $|\mathfrak{b}| R$. The terms on the right-hand sides of Eq. (25) are quadratic forms in $\omega, \epsilon$ and to and their expressions given below were obtained by performing the symbolic operations in Mathematica:

$$
\begin{aligned}
& c^{s}=\chi_{1} b_{b} \cdot b_{b} z, \\
& q^{m s}=\left[\chi_{2} \omega(* \mathfrak{b})+\chi_{3}(* \mathbb{b} \otimes * \mathbb{b}) \mathbf{r}+\chi_{4}(\mathbb{b} \otimes \mathfrak{b}) \mathbf{r}+\chi_{5} \epsilon(* \mathfrak{b})\right] \cdot \mathbf{N} z, \\
& \mathbf{b}^{s}=\chi_{6} \mathfrak{b} \cdot \mathfrak{b b}_{\mathbf{b}} \mathbf{e}+\chi_{7} \omega(* \mathbb{b})+\chi_{8}(* \mathbb{b} \otimes * \mathfrak{b}) \mathbf{r}+\chi_{9}(\mathbb{b} \otimes \mathbb{b}) \mathbf{r}+\chi_{10} \epsilon(* \mathfrak{b}), \\
& \mathbf{f}^{m s}=z\left[\chi_{11} \omega(* \mathbb{b})+\chi_{12}(* \mathbb{b} \otimes * \mathfrak{b}) \mathbf{r}+\chi_{13}(\mathbb{b} \otimes \mathbb{b}) \mathbf{r}+\chi_{14} \epsilon(* \mathfrak{b})\right] \cdot \mathbf{N e} \\
& +\frac{z^{2}}{2}\left[\chi_{15}\left(b_{0} \otimes \mathfrak{b}\right) \mathbf{N}+\chi_{16}\left(b_{b} \cdot b_{b}\right) \mathbf{N}\right]+\left[\chi_{17} \omega \epsilon+\chi_{18} \omega^{2}+\chi_{19} \epsilon^{2}+\chi_{20} \omega(* \mathbb{b}) \cdot \mathbf{r}\right. \\
& \left.+\chi_{21} \epsilon(* \mathbb{b}) \cdot \mathbf{r}+\chi_{22}(* \mathbb{b} \cdot \mathbf{r})(\mathbb{b} \cdot * \mathbf{r})+\chi_{23}(\mathbb{b} \cdot \mathbf{r})^{2}\right] \mathbf{N}, \\
& i^{s}=\chi_{25}(\mathbb{b} \cdot \mathbb{b}) z, \\
& h^{s}=\left[\chi_{26}(\mathbb{b} \otimes \mathbb{b})+\chi_{27}(* \mathbb{b} \otimes * \mathbb{b})\right] \mathbf{r}+\chi_{28} z(\mathbb{b} \cdot \mathbb{b}) \mathbf{e}+\chi_{29} \epsilon(* \mathbb{b})+\chi_{30} \omega(* \mathbb{b}), \\
& \overline{\mathbf{g}}^{s}=z\left[\left(\chi_{31}(\mathbb{b} \otimes \mathbb{b})+\chi_{32}(* \mathbb{b} \otimes * \mathfrak{b})\right) \mathbf{r}+\left(\chi_{33} \epsilon+\chi_{34} \omega\right)(* \mathbb{b})\right] \\
& +\left[\chi_{35}(* \mathfrak{b}) \cdot \mathbf{r}+\chi_{36} \epsilon+\chi_{37} \omega\right](\mathbb{b} \cdot \mathbf{r}) \mathbf{e}, \\
& R^{q s}=\frac{z^{2}}{2} \chi_{38}\left(\mathbb{b} \cdot b_{b}\right) A+\left(\chi_{39} \epsilon^{2}+\chi_{40} \omega^{2}+\chi_{41} \epsilon \omega\right) A+\chi_{42} \mathbf{J}_{A} \cdot(\mathbb{b} \otimes \mathfrak{b}) \\
& +\chi_{43} \mathbf{J}_{A} \cdot(* \mathbb{b} \otimes * b), \\
& R^{f s}=\left[\frac{z^{2}}{2} \chi_{44}\left(b_{b} \cdot b_{b}\right) A+\left(\chi_{47} \epsilon^{2}+\chi_{48} \omega^{2}+\chi_{49} \epsilon \omega\right) A+\chi_{50} \mathbf{J}_{A} \cdot\left(b_{0} \otimes \mathfrak{b}\right)\right. \\
& \left.+\chi_{51} \mathbf{J}_{A} \cdot(* \mathbb{b} \otimes * \mathbb{b})\right] \mathbf{e}+z\left(\chi_{45} \epsilon+\chi_{46} \omega\right) A(* \mathbb{b}), \\
& \overline{\mathbf{R}}^{m s}=\frac{z^{2}}{2}\left(\chi_{52} \epsilon+\chi_{53} \omega\right) A(* \mathfrak{b})+\left(\chi_{55} \epsilon+\chi_{56} \omega\right) \mathbf{J}_{A}(* \mathfrak{b})+z \chi_{54} \mathbf{J}_{A} \cdot(\mathbb{b} \otimes(* \mathfrak{b})) \mathbf{e} .
\end{aligned}
$$

Here $\chi_{1}, \chi_{2}, \ldots, \chi_{56}$ are material constants; their expressions in terms of the elastic constants used in the constitutive relation are given in Appendix A. $A$ equals the area of the cross-section,

$$
\mathbf{J}_{A}=\int_{\mathscr{A}}(* \mathbf{r} \otimes * \mathbf{r}) \mathrm{d} A
$$

is the inertia tensor, vectors $\overline{\mathbf{g}}^{s}$ and $\overline{\mathbf{R}}^{m s}$ are equivalent to the skew symmetric tensors $\mathbf{g}^{\mathrm{s}}$ and $\mathbf{R}^{\mathrm{ms}}$, respectively. 


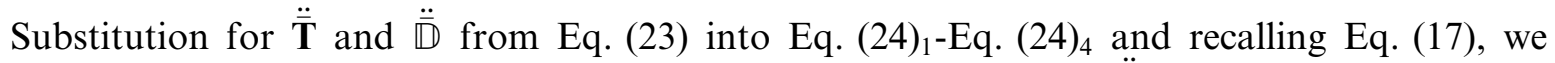
arrive at the following field equations for the determination of $\ddot{\mathbf{u}}$ and $\ddot{\psi}$ :

$$
\begin{aligned}
& F(\ddot{\mathbf{v}})+\left(c_{3}+\lambda+\tilde{\mu}\right) \operatorname{grad} \ddot{w}^{\prime}-\left(e_{2}+e_{3}\right) \operatorname{grad} \ddot{\psi}^{\prime}+\tilde{\mu} \ddot{\mathbf{v}}^{\prime}=\mathbf{b}^{s \mathscr{A}} \text { in } \mathscr{A}, \\
& \Delta_{R} \ddot{\tilde{w}}+\left(c_{3}+\lambda+\tilde{\mu}\right) \operatorname{div} \ddot{\mathbf{v}}^{\prime}+2\left(c_{1}+\frac{\lambda}{2}+c_{3}+c_{4}+\mu\right) \ddot{w}^{\prime \prime} \\
& -\left(e_{1}+e_{2}+2 e_{3}\right) \ddot{\psi}^{\prime \prime}=b^{s e}, \text { in } \mathscr{A}, \\
& \Delta_{R} \ddot{\tilde{\psi}}-\left(e_{2}+e_{3}\right) \operatorname{div} \ddot{\mathbf{v}}^{\prime}+2\left(\epsilon_{1}+\epsilon_{2}-1 / 2\right) \ddot{\psi}^{\prime \prime} \\
& -\left(e_{1}+e_{2}+2 e_{3}\right) \ddot{w}^{\prime \prime}=c^{s}, \text { in } \mathscr{A}, \\
& G(\ddot{\mathbf{v}}) \mathbf{N}+\left[\left(c_{3}+\lambda\right) \ddot{w}^{\prime}-e_{2} \ddot{\psi}^{\prime}\right] \mathbf{N}=f^{m s \mathscr{A}}, \quad \text { on } \partial \mathscr{A}, \\
& \operatorname{grad} \ddot{\tilde{w}} \cdot \mathbf{N}+\tilde{\mu} \ddot{\mathbf{v}}^{\prime} \cdot \mathbf{N}=f^{m s e}, \quad \text { on } \partial \mathscr{A}, \\
& \operatorname{grad} \ddot{\tilde{\psi}} \cdot \mathbf{N}-e_{3} \ddot{\mathbf{v}}^{\prime} \cdot \mathbf{N}=q^{m s}, \quad \text { on } \partial \mathscr{A},
\end{aligned}
$$

where

$$
\begin{aligned}
F(\mathbf{v}) & =\mu \Delta_{R} \mathbf{v}+(\lambda+\mu) \operatorname{grad} \operatorname{div} \mathbf{v}, \\
G(\mathbf{v}) & =2 \mu(\operatorname{grad} \mathbf{v})_{s}+\lambda(\operatorname{div} \mathbf{v}) \hat{\mathbf{I}}, \\
\ddot{\tilde{w}} & =\tilde{\mu} \ddot{w}-e_{3} \ddot{\psi}, \quad \ddot{\tilde{\psi}}=-e_{3} \ddot{w}+\left(2 \epsilon_{2}-1\right) \ddot{\psi}, \\
\mathbf{b}^{s} & =\mathbf{b}^{s \mathscr{A}}+b^{s e} \mathbf{e}, \quad \mathbf{f}^{m s}=\mathbf{f}^{m s \mathscr{A}}+f^{m s e} \mathbf{e},
\end{aligned}
$$

$\Delta_{R}$ is the Laplacian operator and $F=\operatorname{div} G$ is the Navier operator in the cross-section $\mathscr{A}$.

\section{A Saint-Venant/Almansi solution}

We seek a solution of Eq. (29) of the form

$$
\ddot{w}=\sum_{i=0}^{m} \frac{z^{i}}{i !} \ddot{w}_{i}(\mathbf{r}), \quad \ddot{\mathbf{v}}=\sum_{i=0}^{m} \frac{z^{i}}{i !} \ddot{\mathbf{v}}_{i}(\mathbf{r}), \quad \ddot{\psi}=\sum_{i=0}^{m} \frac{z^{i}}{i !} \ddot{\psi}_{i}(\mathbf{r}) .
$$

Substituting from Eq. (32) into Eq. (29), recalling Eq. (28) and equating like powers of $z^{i} / i$ ! on both sides, we obtain partial differential equations, boundary conditions and integrability conditions to determine terms in Eq. (32). For $i>3$, these boundary value problems have null solutions. Henceforth we denote various constants by a superscript zero. For $i=3$, the solution is

$$
\ddot{\mathbf{v}}_{3}=\mathbf{v}_{3}^{0}+\theta_{3}^{0}(* \mathbf{r}), \quad \ddot{w}_{3}=w_{3}^{0}, \ddot{\psi}_{3}=\psi_{3}^{0} .
$$

The integrability conditions for the torque, axial force and the charge require that 


$$
\theta_{3}^{0}=0, \quad w_{3}^{0}=\xi_{3} \mathfrak{b} \cdot \mathbb{b}, \quad \psi_{3}^{0}=\xi_{4} \mathbb{b} \cdot \mathbb{b},
$$

where $\xi_{3}, \xi_{4}$ and other $\xi$ 's introduced below are defined in terms of other elastic constants in Appendix A. With Eqs. (32) and (33), equations for the determination of $\ddot{\mathbf{v}}_{2}, \ddot{\mathbf{w}}_{2}$ and $\ddot{\psi}_{2}$ are

$$
\begin{aligned}
& F\left(\ddot{\mathbf{v}}_{2}\right)=\mathbf{0}, \quad \Delta_{R} \ddot{\tilde{w}}_{2}=0, \quad \Delta_{R} \ddot{\tilde{\psi}}_{2}=0, \quad \text { in } \mathscr{A}, \\
& G\left(\mathbf{v}_{2}\right)=\chi_{15}(\mathbb{b} \otimes \mathbb{b}) \mathbf{N}+\left(\chi_{16}+e_{3} \xi_{4}-\left(c_{3}+\lambda\right) \xi_{3}\right)(\mathbb{b} \cdot \mathbb{b}) \mathbf{N}, \quad \text { on } \partial \mathscr{A}, \\
& \operatorname{grad} \ddot{\tilde{w}}_{2} \cdot \mathbf{N}=-\tilde{\mu} \mathbf{v}_{3}^{0} \cdot \mathbf{N}, \quad \operatorname{grad} \ddot{\tilde{\psi}}_{2} \cdot \mathbf{N}=e_{3} \mathbf{v}_{3}^{0} \cdot \mathbf{N}, \quad \text { on } \partial \mathscr{A},
\end{aligned}
$$

and their solution is

$$
\begin{aligned}
& \ddot{\mathbf{v}}_{2}=\mathbf{v}_{2}^{0}+\theta_{2}^{0}(* \mathbf{r})+\mathbb{B}_{v} \mathbf{r}, \\
& \ddot{w}_{2}=w_{2}^{0}-\mathbf{v}_{3}^{0} \cdot \mathbf{r}, \ddot{\psi}_{2}=\psi_{2}^{0},
\end{aligned}
$$

where

$$
\mathbb{B}_{v}=\xi_{5}(\mathbb{b} \cdot \mathbb{b}) \mathbf{r}+\xi_{1}(\mathbb{b} \otimes \mathbb{b}) \mathbf{r}+\xi_{2}(* \mathbb{b} \otimes * \mathbb{b}) \mathbf{r} .
$$

The integrability conditions for the torque, axial force and the electric charge require that

$$
\begin{aligned}
& \theta_{2}^{0}=\left(\chi_{12}+\chi_{13}+\chi_{35}\right)(\mathbf{J} * \mathbb{b}) \cdot \mathbb{b} /\left(\tilde{\mu} \int_{\mathscr{A}}\left(r^{2}+\operatorname{grad} \phi \cdot * \mathbf{r}\right) \mathrm{d} A\right), \\
& w_{2}^{0}=0, \quad \psi_{2}^{0}=0 .
\end{aligned}
$$

Field equations and boundary conditions for the determination of $\ddot{\mathbf{v}}_{1}$, $\ddot{w}_{1}$ and $\ddot{\psi}_{1}$ are

$$
\begin{aligned}
F\left(\ddot{\mathbf{v}}_{1}\right)= & \left(c_{3}+\lambda\right) \mathbf{v}_{3}^{0}, \Delta_{R} \ddot{\tilde{w}}_{1}=\xi_{6} \mathbb{b} \cdot \mathbb{b}, \Delta_{R} \ddot{\tilde{\psi}}_{1}=\xi_{7} \mathbb{b} \cdot \mathbb{b}, \\
G\left(\ddot{\mathbf{v}}_{1}\right) \mathbf{N}= & \left(c_{3}+\lambda\right)\left(\mathbf{v}_{3}^{0} \cdot \mathbf{r}\right) \mathbf{N}, \\
\operatorname{grad} \ddot{\tilde{w}}_{1} \cdot \mathbf{N}= & -\tilde{\mu}\left(\mathbf{v}_{2}^{0} \cdot \mathbf{N}+\theta_{2}^{0} * \mathbf{r} \cdot \mathbf{N}\right)+\left(\chi_{11} \omega+\chi_{14} \epsilon\right)(* \mathbb{b}) \cdot \mathbf{N}-\tilde{\mu} \xi_{5}(\mathbb{b} \cdot \mathbb{b}) \mathbf{r} \cdot \mathbf{N} \\
& +\left(\chi_{13}-\tilde{\mu} \xi_{1}\right)((\mathbb{b} \otimes \mathbb{b}) \mathbf{r}) \cdot \mathbf{N}+\left(\chi_{12}-\tilde{\mu} \xi_{2}\right)((* \mathbb{b} \otimes * \mathbb{b}) \mathbf{r}) \cdot \mathbf{N}, \\
\operatorname{grad} \ddot{\psi}_{1} \cdot \mathbf{N}= & e_{3} \mathbf{v}_{2}^{0} \cdot \mathbf{N}+e_{3} \theta_{2}^{0}(* \mathbf{r}) \cdot \mathbf{N}+\left(\chi_{2} \omega+\chi_{5} \epsilon\right)(* \mathbb{b}) \cdot \mathbf{N}+e_{3} \xi_{5}((\mathbb{b} \cdot \mathbb{b}) \mathbf{r}) \cdot \mathbf{N} \\
& +\left(\chi_{4}+e_{3} \xi_{1}\right)((\mathbb{b} \otimes \mathbb{b}) \mathbf{r}) \cdot \mathbf{N}+\left(\chi_{3}+e_{3} \xi_{2}\right)((* \mathbb{b} \otimes * \mathbb{b}) \mathbf{r}) \cdot \mathbf{N} .
\end{aligned}
$$

The solution of the boundary-value problem (39) is

$$
\begin{aligned}
& \ddot{\mathbf{v}}_{1}=\mathbf{v}_{1}^{0}+\theta_{1}^{0}(* \mathbf{r})+\tilde{v}(\mathbf{r} \otimes * \mathbf{r})_{s} * \mathbf{v}_{3}^{0}, \\
& \ddot{w}_{1}=w_{1}^{0}-\mathbf{v}_{2}^{0} \cdot \mathbf{r}+\theta_{2}^{0} \phi(\mathbf{r})+\left(\xi_{8} \omega+\xi_{9} \epsilon\right)(* b b) \cdot \mathbf{r}+\left(\mathbb{B}_{w} \mathbf{r}\right) \cdot \mathbf{r}, \\
& \ddot{\psi}_{1}=\psi_{1}^{0}+\left(\xi_{13} \omega+\xi_{14} \epsilon\right) * \mathbb{b} \cdot \mathbf{r}+\left(\mathbb{B}_{\psi} \mathbf{r}\right) \cdot \mathbf{r},
\end{aligned}
$$


where

$$
\begin{aligned}
& \mathbb{B}_{w}=\left[\xi_{10}(\mathbb{b} \otimes \mathbb{b})+\xi_{11}(* \mathbb{b} \otimes * \mathbb{b})+\xi_{12}(\mathbb{b} \cdot \mathbb{b}) \hat{\mathbf{I}}\right], \\
& \mathbb{B}_{\psi}=\left[\xi_{15}(\mathbb{b} \otimes \mathbb{b})+\xi_{16}(* \mathbb{b} \otimes * \mathbb{b})+\xi_{17}(\mathbb{b} \cdot \mathbb{b}) \hat{\mathbf{I}}\right],
\end{aligned}
$$

and $\tilde{v}=\left(c_{3}+\lambda\right) / 2(\lambda+\mu)$ is the Poisson's ratio in the direction of transverse isotropy. Equations for finding fields $\ddot{\mathbf{v}}_{0}, \ddot{w}_{0}$ and $\ddot{\psi}_{0}$ can be written as

$$
\begin{aligned}
F\left(\ddot{\mathbf{v}}_{0}\right)= & \left(c_{3}+\lambda\right) \mathbf{v}_{2}^{0}-\theta_{2}^{0}\left[\left(c_{3}+\lambda+\tilde{\mu}\right) \operatorname{grad} \phi+\tilde{\mu}(* \mathbf{r})\right]+\left(\xi_{18} \omega+\xi_{19} \epsilon\right)(* \mathbb{b})+\mathbb{B} \mathbf{r}, \\
\Delta_{R} \ddot{\tilde{w}}_{0}= & \xi_{22} \mathbf{v}_{3}^{0} \cdot \mathbf{r}, \quad \Delta_{R} \ddot{\tilde{\psi}}_{0}=\xi_{23} \mathbf{v}_{3}^{0} \cdot \mathbf{r}, \\
G\left(\ddot{\mathbf{v}}_{0}\right) \mathbf{N}= & {\left[\left(c_{3}+\lambda\right)\left(\mathbf{v}_{2}^{0} \cdot \mathbf{r}-\theta_{2}^{0} \phi\right)+\chi_{17} \omega \epsilon+\chi_{18} \omega^{2}+\chi_{19} \epsilon^{2}-\left(c_{3}+\lambda\right) w_{1}^{0}+e_{2} \psi_{1}^{0}\right.} \\
& \left.+\left(\xi_{20} \omega+\xi_{21} \epsilon\right) * \mathbb{b} \cdot \mathbf{r}+(\mathbb{B r}) \cdot \mathbf{r} / 2\right] \mathbf{N}+\chi_{24}(\mathbb{b} \cdot \mathbf{r})(* \mathbb{b} \cdot \mathbf{r})(* \mathbf{N}), \\
\left(\operatorname{grad} \ddot{\tilde{w}}_{0}\right) \cdot \mathbf{N}= & -\tilde{\mu}\left[\mathbf{v}_{1}^{0}+\theta_{1}^{0}(* \mathbf{r})+\tilde{v}(\mathbf{r} \otimes * \mathbf{r})_{s} * \mathbf{v}_{3}^{0}\right] \cdot \mathbf{N}, \\
\left(\operatorname{grad} \ddot{\tilde{\psi}}_{0}\right) \cdot \mathbf{N}= & e_{3}\left[\mathbf{v}_{1}^{0}+\theta_{1}^{0}(* \mathbf{r})+\tilde{v}(\mathbf{r} \otimes * \mathbf{r})_{s} * \mathbf{v}_{3}^{0}\right] \cdot \mathbf{N},
\end{aligned}
$$

where

$$
\mathbb{B}=\chi_{8}(* \mathbb{b} \otimes * \mathbb{b})+\chi_{9}(\mathbb{b} \otimes \mathbb{b})-2\left(c_{3}+\lambda+\tilde{\mu}\right) \mathbb{B}_{w}+2\left(e_{2}+e_{3}\right) \mathbb{B}_{\psi}-\tilde{\mu} \mathbb{B}_{v} .
$$

Note that $\mathbb{B}$ is a bilinear form in $\mathbb{b}$ and its eigenvectors are $\mathbb{b}$ and $* \mathbb{b}$. The solution of Eq. (42) is

$$
\begin{aligned}
\ddot{\mathbf{v}}_{0}= & \mathbf{v}_{0}^{0}+\theta_{0}^{0}(* \mathbf{r})+\tilde{v}(\mathbf{r} \otimes * \mathbf{r})_{s}\left(* \mathbf{v}_{2}^{0}\right) \\
& +\frac{\left(\chi_{17} \omega \epsilon+\chi_{18} \omega^{2}+\chi_{19} \epsilon^{2}-\left(c_{3}+\lambda\right) w_{1}^{0}+e_{2} \psi_{1}^{0}\right) \mathbf{r}}{2(\lambda+\mu)} \\
& +\frac{\xi_{18} \omega+\xi_{19} \epsilon}{4(\lambda+\mu)}(\mathbf{r} \otimes \mathbf{r})-(* \mathbf{r} \otimes * \mathbf{r}) * \mathbb{b}+\Theta(\mathbf{r}), \\
\ddot{w}_{0}= & w_{0}^{0}-\mathbf{v}_{1}^{0} \cdot \mathbf{r}+\theta_{1}^{0} \phi(\mathbf{r})+\xi_{26} \Phi(\mathbf{r})+\xi_{27} \Psi(\mathbf{r}), \\
\ddot{\psi}_{0}= & \psi_{0}^{0}-\xi_{27} \Phi(\mathbf{r})+\xi_{28} \Psi(\mathbf{r}),
\end{aligned}
$$

where functions $\Theta, \Phi$ and $\Psi$ are solutions of

$$
\begin{aligned}
F(\Theta) & =-\theta_{2}^{0}\left[\left(c_{3}+\lambda+\tilde{\mu}\right) \operatorname{grad} \phi+\tilde{\mu}(* \mathbf{r})\right]+\mathbb{B} \mathbf{r}, \\
G(\Theta) \mathbf{N} & =-\theta_{2}^{0} \phi \mathbf{N}+\frac{1}{2}(\mathbb{B} \mathbf{r}) \cdot \mathbf{r} \mathbf{N}, \\
\Delta_{R} \Phi & =\xi_{22} \mathbf{v}_{3}^{0} \cdot \mathbf{r}, \operatorname{grad} \Phi \cdot \mathbf{N}=\xi_{24}(\mathbf{r} \otimes * \mathbf{r})_{s}\left(* \mathbf{v}_{3}^{0}\right), \\
\Delta_{R} \Psi & =\xi_{23} \mathbf{v}_{3}^{0} \cdot \mathbf{r}, \operatorname{grad} \Psi \cdot \mathbf{N}=\xi_{25}(\mathbf{r} \otimes * \mathbf{r})_{s}\left(* \mathbf{v}_{3}^{0}\right) .
\end{aligned}
$$

The clamping conditions (6) require that

$$
\mathbf{v}_{0}^{0}=\mathbf{0}, w_{0}^{0}=0, \theta_{0}^{0}=0, \psi_{0}^{0}=0, \mathbf{v}_{1}^{0}=\mathbf{0} .
$$


The second-order solution is specified by seven constants $\mathbf{v}_{3}^{0}, \mathbf{v}_{2}^{0}, \theta_{1}^{0}, w_{1}^{0}$ and $\psi_{1}^{0}$ representing second-order flexure, bending, torsion, elongation and electric potential, respectively.

We now consider the case when the resultant loads are

$$
\mathbf{R}_{F}=\int_{\mathscr{A}} \mathbf{f} \mathrm{d} A=N \mathbf{e}, \quad R_{Q}=\int_{\mathscr{A}} q \mathrm{~d} A=Q, \quad \mathbf{R}_{M}=\int_{\mathscr{A}} \mathbf{x} \wedge \mathbf{f} \mathrm{d} A=M \mathbf{e} \wedge \mathbf{k},
$$

where $\mathbf{k}$ is a unit vector in the cross-section $\mathscr{A}$. For these loads,

$$
\epsilon=\xi_{29} N+\xi_{30} Q, \quad \omega=\xi_{30} N+\xi_{31} Q, \quad \mathbb{b}=-\tilde{E} M \mathbf{J}_{A}^{-1}(* \mathbf{k}),
$$

where $\tilde{E}$ is the Young's modulus in the direction of transverse isotropy; an expression for $\tilde{E}$ in terms of other material constants is given in the Appendix (see the line between expressions for $\xi_{31}$ and $\xi_{32}$ ). For loads given by Eq. (47)

$$
\begin{aligned}
\mathbf{v}(\mathbf{r}, z)= & \tilde{v}\left(1+\xi_{32} \epsilon+\xi_{33} \omega\right)(\mathbf{r} \otimes * \mathbf{r})_{s} \mathbb{b}+\left[-v(a \omega+\epsilon)+\xi_{34} \omega \epsilon+\xi_{35} \epsilon^{2}\right. \\
& \left.+\xi_{36} \omega^{2}+(\mathbf{J} / A) \cdot\left(\xi_{38} \mathbb{b} \otimes \mathbb{b}+\xi_{39} * \mathbb{b} \otimes * \mathbb{b}\right)\right] \mathbf{r}-\frac{z^{2}}{2}\left(1+\tilde{v}\left(\xi_{32} \epsilon+\xi_{33} \omega\right)\right) * \mathbb{b} \\
& +\frac{\left(\xi_{18} \omega+\xi_{19} \epsilon\right)}{4(\lambda+\mu)}(\mathbf{r} \otimes \mathbf{r}-* \mathbf{r} \otimes * \mathbf{r}) * \mathbb{b}+\Theta(\mathbf{r})+\frac{z^{2}}{2}\left(\theta_{2}^{0} * \mathbf{r}+\mathbb{B}_{v} \mathbf{r}\right), \\
w(r, z)= & z\left[a \omega+\epsilon-v\left(\xi_{35} \epsilon^{2}+\xi_{34} \epsilon \omega+\xi_{36} \omega^{2}\right)-\tilde{v} \frac{\mathbf{J}}{A} \cdot\left(\xi_{38} \mathbb{b} \otimes \mathbb{b}+\xi_{39} * \mathbb{b} \otimes * \mathbb{b}\right)\right] \\
& +z * \mathbb{b} \cdot \mathbf{r}\left(1+\tilde{v} \xi_{32} \epsilon+\tilde{v} \xi_{33} \omega\right)+z\left[\theta_{2}^{0} \phi(\mathbf{r})+\mathbb{B}_{w} \mathbf{r} \cdot \mathbf{r}\right]+\frac{z^{3}}{6} \xi_{3} \mathbb{b} \cdot \mathbb{b}, \\
\psi(r, z)= & z\left[\omega+b \epsilon-v\left(b^{2} \xi_{35} \epsilon^{2}+\xi_{34} \epsilon \omega+\xi_{36} a^{2} \omega^{2}\right)-\tilde{v}(b \mathbf{J} / A) \cdot\left(\xi_{38} \mathbb{b} \otimes \mathbb{b}\right.\right. \\
& \left.\left.+\xi_{39} * \mathbb{b} \otimes * \mathbb{b}\right)+\mathbb{B}_{\psi} \mathbf{r} \cdot \mathbf{r}\right]+\frac{z^{3}}{6} \xi_{4} \mathbb{b} \cdot \mathbb{b} .
\end{aligned}
$$

\section{Discussion of results}

We now observe a few interesting features of the solution. When $N=Q=0$ and $M \neq 0$, i.e., the end faces of the bar are subjected to a pure bending moment, we conclude from Eq. (48) that $\epsilon=\omega=0$ and from Eq. (49) we get a second-order Poisson's effect proportional to $\|$ b $\|^{2}$ and a torsional effect proportional to the square of the axial distance from the end face whose centroid is clamped. The term $\left(z^{2} / 2\right) \mathbb{B}_{v} \mathbf{r}$ implies that a circular cross-section will be deformed into an ellipse and this distortion of the cross-section is proportional to the square of the distance from the 'clamped' end face. In addition to the classical axial strain in the linear theory, there is an axial strain which depends quadratically upon tb and also on $z^{2}$. For an isotropic material $\xi_{3}=-1$ and our results reduce to those of dell'Isola et al. [19]. The term $z \theta_{2}^{0} \phi(\mathbf{r})$ in Eq. (49) $)_{2}$ is associated with the torsional effect mentioned above. The potential difference 
between the end faces is proportional to the cube of the length and is quadratic in th. The term multiplying $z$ in Eq. (49) $)_{3}$ can vanish for some cross-sections but that multiplying $z^{3}$ is always non-zero. Even if the magnitude of $\xi_{4}$ is small, one can enhance the potential difference by increasing the length of the bar. One can determine the direction of bending because of the term $z \mathbb{B}_{\psi} \mathbf{r} \cdot \mathbf{r}$ and the eigenvectors of $\mathbb{B}_{\psi}$ are $\mathbb{b}$ and $* \mathfrak{b}$. For an arbitrary choice of the values of material parameters and a circular cross-section, Fig. 1 depicts the electric potential generated by the term $z \mathbb{B}_{\psi} \mathbf{r} \cdot \mathbf{r}$ for $z=1$.

When $M=0, \mathbb{b}=\mathbf{0}$ but $\omega$ and $\epsilon$ need not vanish. In this case we get second-order Poisson's effect, axial strain and potential difference between the end faces which are the same for every cross-section. Even for a second-order piezoelectric material, the electric field does not induce any bending of the bar.

The loadings $M \neq 0$ and $Q \neq 0$ result in interesting coupling effects between bending and electric polarization. The curvature of the centroidal axis is given by

$$
\left.\mathbf{v}^{\prime \prime}\right|_{\mathbf{r}=\mathbf{0}}=* \mathbb{b}\left(1+\tilde{v}\left(\xi_{32} \epsilon+\xi_{33} \omega\right)\right) .
$$

Thus the electric field influences the curvature of a prebent bar. The Poisson effect and the axial strain associated with this are represented by the terms $\tilde{v}(\mathbf{r} \otimes * \mathbf{r})_{s}\left(\xi_{32} \epsilon+\xi_{33} \omega\right)$ b), $\tilde{v}\left(\xi_{32} \epsilon+\xi_{33} \omega\right) *$ $\llbracket b \cdot \mathbf{r}$ in Eq. (49) $)_{1}$ and Eq. (49) $)_{2}$, respectively.

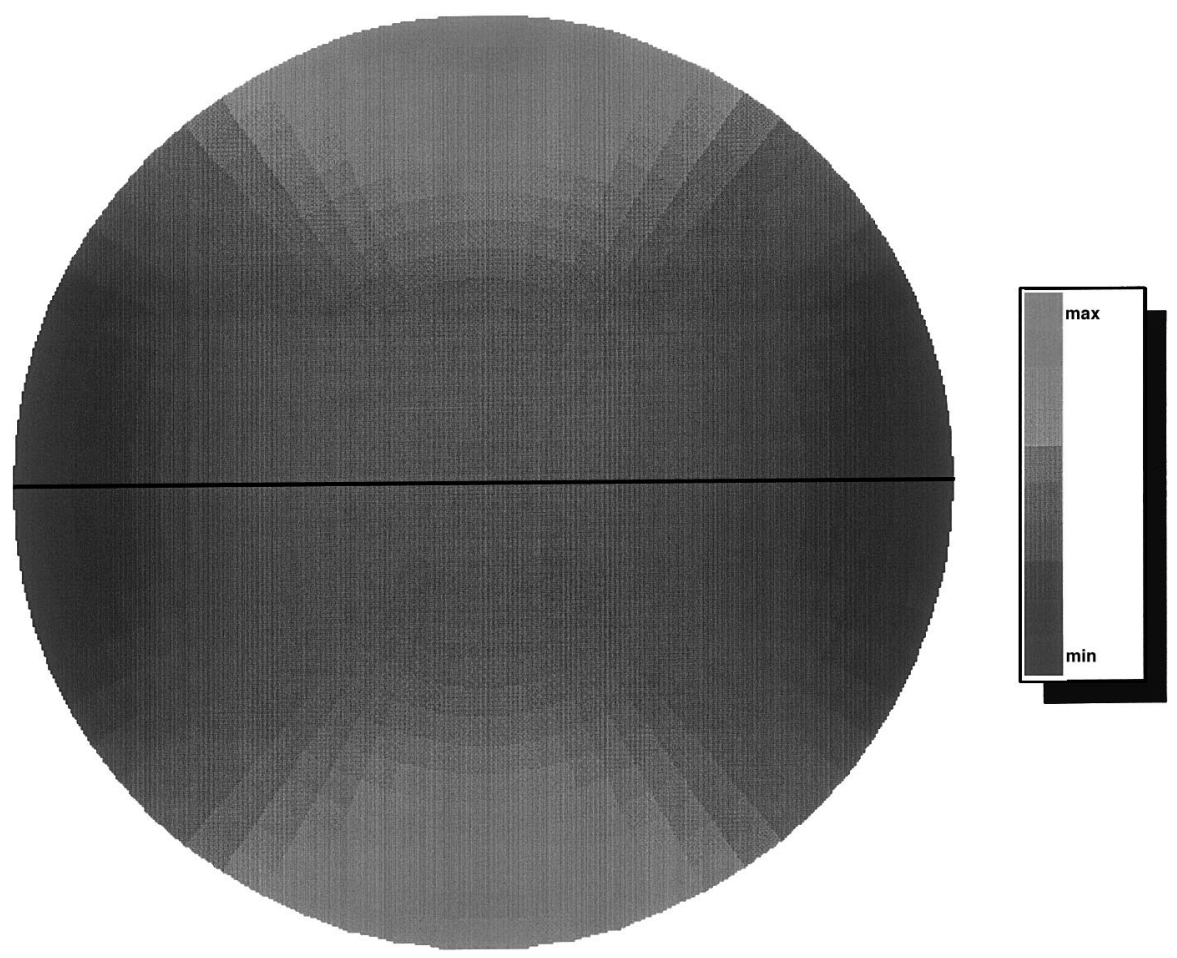

Fig. 1. The electric potential generated by $\mathbb{b}_{\psi} \mathbf{r} \cdot \mathbf{r}$ in a circular bar bent about the horizontal axis. 
For a prismatic bar of circular cross-section $\mathbf{J}$ is a spherical tensor, $(\mathbf{J} * \mathbb{b}) \cdot \mathbb{b}=0$ and from Eq. $(38)_{1}$ we obtain $\theta_{2}^{0}=0$. The boundary-value problem $(45)_{1}$ and $(45)_{2}$ for the determination of $\Theta$ simplifies to

$$
\begin{aligned}
& \mu \Delta_{R} \Theta+(\lambda+\mu) \operatorname{grad} \operatorname{div} \Theta=\mathbb{B} \mathbf{r} \text { in } \mathscr{A} \\
& {\left[2 \mu(\operatorname{grad} \Theta)_{s}+\lambda(\operatorname{div} \Theta) \hat{\mathbf{I}}\right] \mathbf{N}=\frac{R^{2}}{2}(\mathbb{B N} \cdot \mathbf{N}) \mathbf{N}, \quad \text { on } \mathscr{A}}
\end{aligned}
$$

For the bending vector bo directed along a principal axis of inertia of a rectangular cross-section, the boundary-value problem $(45)_{1}$ and $(45)_{2}$ for $\Theta$ can also be somewhat simplified.

\section{Conclusions}

We have found a second-order solution for the Saint-Venant problem for a straight, prismatic, homogeneous and transversely isotropic body made of a second-order piezoelectric material. The bar is stress and polarization free in the reference configuration. It is initially bent, stretched and electrically polarized by an infinitesimal amount and then deformed by loads and electric potential applied to its end faces. The displacements, infinitesimal rotations and the electric potential are assumed to vanish at the centroid of one end face to eliminate trivial solutions of the problem.

It is found that there is a second-order effect, not of the Saint-Venant type, which is quadratic in the bending vector $\mathbb{b}$, electric field $\omega$ and the axial elongation $\epsilon$ and varies as $r^{2}$ where $r$ is the distance of the point from the centroidal axis. The in-plane displacements represented by $\Theta$ in Eq. (49) $)_{1}$ can not be characterized unless the plane elliptic problem defined by Eq. (45) $)_{1}$ and Eq. $(45)_{2}$ has been solved.

For a prismatic circular bar subjected to bending moments only at the end faces, in addition to the second-order Poisson's effect proportional to $\|\mathbb{b}\|^{2}$, there is also a torsional effect proportional to the square of the distance from the 'clamped' face. The circular cross-section is deformed into an ellipse and the distortion of the cross-section is proportional to the square of the distance from the 'clamped' end. The potential difference between the end faces of a prebent bar is proportional to the cube of its length and is quadratic in $\mathbb{b}$.

When a bending moment and an electric charge are simultaneously applied to the end faces of a prismatic bar, then the curvature of its centroidal axis is altered by the electric field.

\section{Acknowledgements}

R.C. Batra's and S. Vidoli's work was partially supported by the NSF grant CMS9713453 and the ARO grant DAAG55-98-1-0030 to Virginia Polytechnic Institute and State University. F. dell'Isola thanks Prof. N. Rizzi for having secured financial support for his visit to Virginia Tech. Many of the algebraic operations were performed by using Mathematica. 


\section{Appendix A}

Using the notations

$$
\begin{aligned}
& \dot{\mathbf{E}}=(\dot{\mathbf{H}}+\dot{\mathbf{H}})^{T} / 2, \quad \ddot{\mathbf{E}}=\dot{\mathbf{H}}^{T} \dot{\mathbf{H}} / 2, \\
& \dot{I}_{1}=\mathbf{e} \cdot(\dot{\mathbf{E} e}), \quad \ddot{I}_{1}=\mathbf{e} \cdot(\ddot{\mathbf{E}} \mathbf{e}), \quad \dot{I}_{2}=\operatorname{tr} \dot{\mathbf{H}}, \ddot{I}_{2}=\operatorname{tr} \ddot{\mathbf{E}}, \\
& \dot{I}_{3}=\dot{\mathbf{W}} \cdot \mathbf{e}, \quad \ddot{\Pi}_{1}=\mathbf{e} \cdot\left(\dot{\mathbf{E}}^{2} \mathbf{e}\right), \quad \ddot{\Pi}_{2}=(\operatorname{tr} \dot{\mathbf{E}})^{2}, \quad \ddot{\Pi}_{3}=\dot{\mathbf{W}} \cdot \dot{\mathbf{W}}, \ddot{\Pi}_{4}=\mathbf{e} \cdot(\dot{\mathbf{E}} \dot{\mathbf{W}})+\dot{\mathbf{W}} \cdot(\dot{\mathbf{E}} \mathbf{e}),
\end{aligned}
$$

constitutive relations for second-order transversely isotropic material with the axis of transverse isotropy along the unit vector e are as follows:

$$
\begin{aligned}
& \dot{\mathbf{T}}=\left(2 c_{1} \dot{I}_{1}+c_{3} \dot{I}_{2}+e_{1} \dot{I}_{3}\right) \mathbf{e} \otimes \mathbf{e}+\left(2 c_{2} \dot{I}_{2}+c_{3} \dot{I}_{1}+e_{2} \dot{I}_{3}\right) \mathbf{1} \\
& +c_{4}(\mathbf{e} \otimes \dot{\mathbf{E}} \mathbf{e})_{s}+2 c_{5} \dot{\mathbf{E}}+e_{3}(\mathbf{e} \otimes \dot{\mathbf{W}}), \\
& \ddot{\mathbf{T}}=\left[2 c_{1} \ddot{I}_{1}+c_{3} \ddot{I}_{2}+3 \lambda_{1} \dot{I}_{1}^{2}+2 \lambda_{3} \dot{I}_{1} \dot{I}_{2}+\lambda_{4} \dot{I}_{2}^{2}+\lambda_{5} \ddot{\Pi}_{1}\right. \\
& \left.+\lambda_{7} \ddot{\Pi}_{2}+2 v_{1} \dot{I}_{1} \dot{I}_{3}+v_{2} \dot{I}_{3}^{2}+v_{7} \ddot{\Pi}_{3}+v_{9} \ddot{\Pi}_{4}+v_{14} \dot{I}_{2} \dot{I}_{3}\right] \mathbf{e} \otimes \mathbf{e} \\
& +\left[2 c_{2} \ddot{I}_{2}+c_{3} \ddot{I}_{1}+3 \lambda_{2} \dot{I}_{2}^{2}+\lambda_{3} \dot{I}_{1}^{2}+2 \lambda_{4} \dot{I}_{1} \dot{I}_{2}+\lambda_{6} \ddot{\Pi}_{1}+\lambda_{8} \ddot{\Pi}_{2}\right. \\
& \left.+2 v_{3} \dot{I}_{2} \dot{I}_{3}+v_{4} \dot{I}_{3}^{2}+v_{8} \ddot{\Pi}_{3}+v_{10} \ddot{\Pi}_{4}+v_{14} \dot{I}_{1} \dot{I}_{3}\right] \mathbf{1} \\
& +2 c_{4}(\mathbf{e} \otimes \dot{\mathbf{E}} \mathbf{e})_{s}+2\left(\lambda_{5} \dot{I}_{1}+\lambda_{6} \dot{I}_{2}+v_{5} \dot{I}_{3}\right)(\mathbf{e} \otimes \dot{\mathbf{E}} \mathbf{e})_{s} \\
& +2 c_{5} \ddot{\mathbf{E}}+2\left(\lambda_{7} \dot{I}_{1}+\lambda_{8} \dot{I}_{2}+v_{6} \dot{I}_{3}\right) \dot{\mathbf{E}}+2\left(v_{9} \dot{I}_{1}+v_{10} \dot{I}_{2}+v_{11} \dot{I}_{3}\right)(\mathbf{e} \otimes \dot{\mathbf{W}})_{s} \\
& +3 \lambda_{9}(\dot{\mathbf{E}})^{2}+v_{12} \dot{\mathbf{W}} \otimes \dot{\mathbf{W}}+2 v_{13}(\mathbf{e} \otimes \dot{\mathbf{E}} \dot{\mathbf{W}}+\dot{\mathbf{W}} \otimes \dot{\mathbf{E}} \mathbf{e})_{s} \\
& +\dot{\mathbf{H}}\left\{\left(2 c_{1} \dot{I}_{1}+c_{3} \dot{I}_{2}+e_{1} \dot{I}_{3}\right) \mathbf{e} \otimes \mathbf{e}+\left(2 c_{2} \dot{I}_{2}+c_{3} \dot{I}_{1}+e_{2} \dot{I}_{3}\right) \mathbf{1}\right. \\
& \left.+c_{4}(\mathbf{e} \otimes \dot{\mathbf{E}} \mathbf{e})_{s}+2 c_{5} \dot{\mathbf{E}}+e_{3}(\mathbf{e} \otimes \dot{\mathbf{W}})_{s}\right\}, \\
& \dot{\mathbf{D}}=-\left(2 \epsilon_{1} \dot{I}_{3}+e_{1} \dot{I}_{1}+e_{2} \dot{I}_{2}\right) \mathbf{e}+\left(1-2 \epsilon_{2}\right) \dot{\mathbf{W}}-2 e_{3} \dot{\mathbf{E}} \mathbf{e}, \\
& \ddot{\mathbf{D}}=\left(1-2 \epsilon_{2}\right) \ddot{\mathbf{W}}+\dot{I}_{2} \dot{\mathbf{H}}-2 \dot{\mathbf{E}} \dot{\mathbf{W}}-\left[2 \epsilon_{1} \ddot{I}_{3}+e_{1} \ddot{I}_{1}+e_{2} \ddot{I}_{2}+3 \mu_{1} \dot{I}_{3}^{2}+\mu_{2} \ddot{\Pi}_{3}+v_{1} \dot{I}_{1}^{2}+2 v_{2} \dot{I}_{3} \dot{I}_{1}+v_{3} \dot{I}_{2}^{2}\right. \\
& \left.+2 v_{4} \dot{I}_{3} \dot{I}_{2}+v_{5} \ddot{\Pi}_{1}+v_{6} \ddot{\Pi}_{2}+v_{11} \ddot{\Pi}_{4}+v_{14} \dot{I}_{1} \dot{I}_{2}\right] \mathbf{e} \\
& -2\left[\mu_{2} \dot{I}_{3}+v_{7} \dot{I}_{1}+v_{8} \dot{I}_{2}\right] \dot{\mathbf{W}}-2 e_{3} \ddot{\mathbf{E}} \mathbf{e}-2\left(v_{9} \dot{I}_{1}+v_{10} \dot{I}_{2}+v_{11} \dot{I}_{3}\right) \dot{\mathbf{E}} \mathbf{e}-2 v_{12} \dot{\mathbf{E}} \dot{\mathbf{W}}-2 v_{13} \ddot{\mathbf{E}}^{2} \mathbf{e} .
\end{aligned}
$$

Here $c_{1}, c_{2}, \ldots, e_{1}, e_{2}, \ldots, \lambda_{1}, \lambda_{2}, \ldots, v_{1}, v_{2}, \ldots, \epsilon_{1}, \epsilon_{2}, \ldots$, and $\mu_{1}, \mu_{2}, \ldots$ are material parameters. Expressions for $\chi_{1}, \chi_{2} \ldots$ and $\xi_{1}, \xi_{2}, \ldots$ in terms of these material parameteres are given below, and $b^{\wedge} c$ equals $b$ raised to the power $c$.

$$
\begin{aligned}
& \chi_{1}=e_{1}+2 e_{2}+3 e_{3}, \\
& \chi_{2}=\left((3 \lambda+2 \mu) e_{2} e_{3}\right) /\left(2(\lambda+\mu) c_{3}\right), \\
& \chi_{3}=\left((3 \lambda+2 \mu) e_{3}\right) /(2(\lambda+\mu)), \\
& \chi_{4}=-\left(\lambda e_{3}\right) /(2(\lambda+\mu)), \\
& \chi_{5}=\left((3 \lambda+2 \mu) e_{3}\right) /(2(\lambda+\mu)),
\end{aligned}
$$




$$
\begin{aligned}
& \chi_{6}=-2 \lambda-3 \mu-2 c_{1}-4 c_{3}-\left(5 c_{4}\right) / 2, \\
& \chi_{7}=-1 /\left(4(\lambda+\mu) \wedge 2 c_{3}\right) e_{2}(6 \lambda \wedge 3+4 \lambda \wedge 2 \mu \\
& -6 \lambda \mu \wedge 2-4 \mu \wedge 3-8(\lambda+\mu) \wedge 2 c_{1}-5 \lambda \wedge c_{4}-11 \lambda \mu c_{4}-6 \mu \wedge 2 c_{4} \\
& +24 \mu \wedge 2 \lambda_{2}+8 \lambda \wedge 2 \lambda_{3}+16 \lambda \mu \lambda_{3}+8 \mu \wedge 2 \lambda_{3}+16 \lambda \mu \lambda_{4}+16 \mu \wedge 2 \lambda_{4}+8 \lambda \wedge 2 \lambda_{6}+16 \lambda \mu \lambda_{6} \\
& \left.+8 \mu \wedge 2 \lambda_{6}-8 \lambda \wedge 2 \lambda_{7}-8 \lambda \mu \lambda_{7}+12 \lambda \wedge 2 \lambda_{8}+8 \lambda \mu \lambda_{8}+8 \mu \wedge 2 \lambda_{8}+6 \lambda \wedge 2 \lambda_{9}\right)+2(\lambda+\mu) \\
& \times c_{3}\left(2(\lambda+\mu) e_{1}+\lambda e_{2}+4 \lambda e_{3}+4 \mu e_{3}-4 \mu v_{3}+2 \lambda v_{6}-4 \lambda v_{10}-4 \mu v_{10}-2 \lambda v_{14}-2 \lambda v_{14}\right), \\
& \chi_{8}=-1 /(4(\lambda+\mu) \wedge 2)(6 \lambda \wedge 3+4 \lambda \wedge 2 \mu \\
& -6 \lambda \mu \wedge 2-4 \mu \wedge 3-8(\lambda+\mu) \wedge 2 c_{1}-2(\lambda \wedge 2+3 \lambda \mu+2 \mu \wedge 2) c_{3} \\
& -5 \lambda \wedge 2 c_{4}-11 \lambda \mu c_{4}-6 \mu \wedge 2 c_{4}+24 \mu \wedge 2 \lambda_{2}+8 \lambda \wedge 2 \lambda_{3}+16 \lambda \mu \lambda_{3}+8 \mu \wedge 2 \lambda_{3}+16 \lambda \mu \lambda_{4} \\
& +16 \mu \wedge 2 \lambda_{4}+8 \lambda \wedge 2 \lambda_{6}+16 \lambda_{\mu} \lambda_{6}+8 \mu \wedge 2 \lambda_{6}-8 \lambda \wedge 2 \lambda_{7}-8 \lambda_{\mu} \lambda_{7}+12 \lambda \wedge 2 \lambda_{8} \\
& \left.+8 \lambda \mu \lambda_{8}+8 \mu \wedge 2 \lambda_{8}+6 \lambda \wedge 2 \lambda_{9}\right), \\
& \chi_{9}=\left(\lambda\left(-2 \lambda+2 \mu+2 c_{3}=c_{4}\right)\right) /(4(\lambda+\mu)), \\
& \chi_{10}=-1 /\left(4(\lambda+\mu) \wedge 2 e_{2}\right)\left(e_{2}(6 \lambda \wedge 3+4 \lambda \wedge 2 \mu\right. \\
& -6 \lambda \mu \wedge 2-4 \mu \wedge 3-8(\lambda+\mu) \wedge 2 c_{1}-5 \lambda \wedge 2 c_{4}-11 \lambda \mu c_{4} \\
& -6 \mu \wedge 2 c_{4}+24 \mu \wedge 2 \lambda_{2}+8 \lambda \wedge 2 \lambda_{3}+16 \lambda \mu \lambda_{3}+8 \mu \wedge 2 \lambda_{3}+16 \lambda \mu \lambda_{4}+16 \mu \wedge 2 \lambda_{4} \\
& \left.+8 \lambda \wedge 2 \lambda_{6}+16 \lambda \mu \lambda_{6}+8 \mu \wedge 2 \lambda_{6}-8 \lambda \wedge 2 \lambda_{7}-8 \lambda \mu \lambda_{7}+12 \lambda \wedge 2 \lambda_{8}+8 \mu \wedge 2 \lambda_{8}+6 \lambda \wedge 2 \lambda_{9}\right) \\
& \left.+\lambda e_{2}+4 \lambda e_{3}+4 \mu e_{3}-4 \mu v_{3}+2 \lambda v_{6}-4 \lambda v_{10}-4 \mu v_{10}-2 \lambda v_{14}-2 \mu v_{14}\right) \text {, } \\
& \chi_{11}=-\left((3 \lambda+2 \mu)\left(2 \mu+c_{4}\right) e_{2}\right) /\left(4(\lambda+\mu) c_{3}\right), \\
& \chi_{12}=-\left(4(\lambda+\mu) c_{3}+(3 \lambda+2 \mu)\left(2 \mu+c_{4}\right)\right) /(4(\lambda+\mu)) \text {, } \\
& \chi_{13}=\left(\lambda\left(2 \mu+c_{4}\right)\right) /(4(\lambda+\mu)), \\
& \chi_{14}=-\left((3 \lambda+2 \mu)\left(2 \mu+c_{4}\right)\right) /(4(\lambda+\mu)), \\
& \chi_{15}=2 \mu, \\
& \chi_{16}=-2 \lambda-2 \mu-c_{3}, \\
& \chi_{17}=-1 /\left(2(\lambda+\mu) \wedge 2 c_{3} e_{2}\right) e_{2}^{2}(3 \lambda \wedge 3+5 \lambda \wedge 2 \mu \\
& +2 \lambda \mu \wedge 2+12 \mu \wedge 2 \lambda_{2}+4 \lambda \wedge 2 \lambda_{3}+8 \lambda \mu \lambda_{3}+4 \mu \wedge 2 \lambda_{3}+8 \lambda \mu \lambda_{4} \\
& +8 \mu \wedge 2 \lambda_{4}+4 \lambda \wedge 2 \lambda_{6}+8 \lambda \mu \lambda_{6}+4 \mu \wedge 2 \lambda_{6}-4 \lambda \wedge 2 \lambda_{7}-4 \lambda \mu \lambda_{7}+6 \lambda \wedge 2 \lambda_{8}+4 \lambda \mu \lambda_{8} \\
& \left.+4 \mu \wedge 2 \lambda_{8}+3 \lambda \wedge 2 \lambda_{9}\right)+2(\lambda+\mu) \wedge 2 c_{3}^{2}\left(-1+2 v_{4}+2 v_{8}\right) \\
& +2(\lambda+\mu) c_{3} e_{2}\left((\lambda+\mu) e_{2}-2\left(2 \mu v_{3}-\lambda v_{6}+2 \lambda v_{10}+2 \mu v_{10}+\lambda v_{14}+\mu v_{14}\right)\right),
\end{aligned}
$$




$$
\begin{aligned}
& \chi_{18}=-1 /\left(4(\lambda+\mu) \wedge 2 c_{3}^{2}\right)\left(e_{2}^{2}(3 \lambda \wedge 3+5 \lambda \wedge 2 \mu\right. \\
& +2 \lambda \mu \wedge 2+12 \mu \wedge 2 \lambda_{2}+4 \lambda \wedge 2 \lambda_{3}+8 \lambda \mu \lambda_{3}+4 \mu \wedge 2 \lambda_{3}+8 \lambda \mu \lambda_{4} \\
& +8 \mu \wedge 2 \lambda_{4}+4 \lambda \wedge 2 \lambda_{6}+8 \lambda \mu \lambda_{6}+4 \mu \wedge 2 \lambda_{6}-4 \lambda \mu \lambda_{7}-4 \lambda \mu \lambda_{7}+6 \lambda \wedge 2 \lambda_{8}+4 \lambda \mu \lambda_{8} \\
& \left.+4 \mu \wedge 2 \lambda_{8}+3 \lambda \wedge 2 \lambda_{9}\right) 2(\lambda+\mu) \wedge 2 c_{3}^{2}\left(-1+2 v_{4}+2 v_{8}\right) \\
& \left.+2(\lambda+\mu) c_{3} e_{2}\left((\lambda+\mu) e_{2}-2\left(2 \mu v_{3}-\lambda v_{6}+2 \lambda v_{10}+2 \mu v_{10}+\lambda v_{14}+\mu v_{14}\right)\right)\right), \\
& \chi_{19}=-1 /\left(4(\lambda+\mu) \wedge 2 e_{2}^{2}\right)\left(e_{2}^{2}(3 \lambda \wedge 3+5 \lambda \wedge 2 \mu\right. \\
& +2 \lambda \mu \wedge 2+12 \mu \wedge 2 \lambda_{2}+4 \lambda \wedge 2 \lambda_{3}+8 \lambda \mu \lambda_{3}+4 \mu \wedge 2 \lambda_{3}+8 \lambda \mu \lambda_{4} \\
& +8 \mu \wedge 2 \lambda_{4}+4 \lambda \wedge 2 \lambda_{6}+8 \lambda \mu \lambda_{6}+4 \mu \wedge 2 \lambda_{6}-4 \lambda \wedge 2 \lambda_{7}-4 \lambda \mu \lambda_{7}+6 \lambda \wedge 2 \lambda_{8}+4 \lambda \mu \lambda_{8} \\
& \left.+4 \mu \wedge 2 \lambda_{8}+3 \lambda \wedge 2 \lambda_{9}\right)+2(\lambda+\mu) \wedge 2 c_{3}^{2}\left(-1+2 v_{4}+2 v_{8}\right) \\
& \left.+2(\lambda+\mu) c_{3} e_{2}\left((\lambda+\mu) e_{2}-2\left(2 \mu v_{3}-\lambda v_{6}+2 \lambda v_{10}+2 \mu v_{10}+\lambda v_{14}+\mu v_{14}\right)\right)\right), \\
& \chi_{20}=-1 /\left(2(\lambda+\mu) \wedge 2 c_{3}\right)\left(e _ { 2 } \left(3 \lambda \wedge 3+5 \lambda \wedge 2 \mu+2 \lambda \mu \wedge 2+(\lambda \wedge 2+3 \lambda \mu+2 \mu \wedge 2) c_{3}\right.\right. \\
& +12 \mu \wedge 2 \lambda_{2}+4 \wedge 2 \lambda_{3}+8 \lambda \mu \lambda_{3}+4 \mu \wedge 2 \lambda_{3}+8 \lambda \mu \lambda_{4}+8 \mu \wedge 2 \lambda_{4}+4 \lambda \wedge 2 \lambda_{6} \\
& \left.+8 \lambda \mu \lambda_{6}+4 \mu \wedge 2 \lambda_{6}-4 \lambda \wedge 2 \lambda_{7}-4 \lambda \mu \lambda_{7}+6 \lambda \wedge 2 \lambda_{8}+4 \lambda \mu \lambda_{8}+4 \mu \wedge 2 \lambda_{8}+3 \lambda \wedge 2 \lambda_{9}\right) \\
& \left.+2(\lambda+\mu) c_{3}\left(-2 \mu v_{3}+\lambda v_{6}-2 \lambda v_{10}-2 \mu v_{10}-\lambda v_{14}-\mu v_{14}\right)\right), \\
& \chi_{21}=-1 /\left(2(\lambda+\mu) \wedge 2 e_{2}\right)\left(e _ { 2 } \left(3 \lambda \wedge 3+5 \lambda \wedge 2 \mu+2 \lambda \mu \wedge 2+(\lambda \wedge 2+3 \lambda \mu+2 \mu \wedge 2) c_{3}\right.\right. \\
& +12 \mu \wedge 2 \lambda_{2}+4 \wedge 2 \lambda_{3}+8 \lambda \mu \lambda_{3}+4 \mu \wedge 2 \lambda_{3}+8 \lambda \mu \lambda_{4}+8 \mu \wedge 2 \lambda_{4}+4 \lambda \wedge 2 \lambda_{6} \\
& \left.+8 \lambda \mu \lambda_{6}+4 \mu \wedge 2 \lambda_{6}-4 \lambda \wedge 2 \lambda_{7}-4 \lambda \mu \lambda_{7}+6 \lambda \wedge 2 \lambda_{8}+4 \lambda \mu \lambda_{8}+4 \mu \wedge 2 \lambda_{8}+3 \lambda \wedge 2 \lambda_{9}\right) \\
& \left.+2(\lambda+\mu) c_{3}\left(-2 \mu v_{3}+\lambda v_{6}-2 \lambda v_{10}-2 \mu v_{10}-\lambda v_{14}-\mu v_{14}\right)\right), \\
& \chi_{22}=1 /(4(\lambda+\mu) \wedge 2)\left(3 \lambda \wedge 3+5 \lambda \wedge 2 \mu+2 \lambda \mu \wedge 2+2 \mu(\lambda+\mu) c_{3}\right. \\
& +12 \mu \wedge 2 \lambda_{2}+4 \lambda \wedge 2 \lambda_{3}+8 \lambda \mu \lambda_{3}+4 \mu \wedge 2 \lambda_{3}+8 \lambda \mu \lambda_{4}+8 \mu \wedge 2 \lambda_{4}+4 \lambda \wedge 2 \lambda_{6} \\
& \left.+8 \lambda \mu \lambda_{6}+4 \mu \wedge 2 \lambda_{6}-4 \lambda \wedge 2 \lambda_{7}-4 \lambda \mu \lambda_{7}+6 \lambda \wedge 2 \lambda_{8}+4 \lambda \mu \lambda_{8}+4 \mu \wedge 2 \lambda_{8}+3 \lambda \wedge 2 \lambda_{9}\right), \\
& \chi_{23}=-\lambda \wedge 2 /(4(\lambda+\mu)) \text {, } \\
& \chi_{25}=e_{1}+2\left(e_{2}+e_{3}\right) \text {, } \\
& \chi_{26}=\left(\lambda\left(2 \mu+c_{4}\right)\right) /(4(\lambda+\mu)) \text {, } \\
& \chi_{27}=\left(6 \lambda \mu+4 \mu \wedge 2+8(\lambda+\mu) c_{1}+4(\lambda+2 \mu) c_{3}+5 \lambda c_{4}+6 \mu c_{4}\right) /(4(\lambda+\mu)) \text {, } \\
& \chi_{28}=-2 c_{1}-3 c_{3}-2\left(\lambda+\mu+c_{4}\right) \text {, } \\
& \chi_{29}=1 /\left(4(\lambda+\mu) e_{2}\right)\left(\left(6 \lambda \mu+4 \mu \wedge 2+8(\lambda+\mu) c_{1}\right.\right. \\
& \left.\left.+5 \lambda c_{4}+6 \mu c_{4}\right) e_{2}-4 c_{3}\left((\lambda+\mu) e_{1}-\mu e_{2}+2 \lambda e_{3}+2 \mu e_{3}\right)\right),
\end{aligned}
$$




$$
\begin{aligned}
& \chi_{30}=1 /\left(4(\lambda+\mu) c_{3}\right)\left(\left(6 \lambda \mu+4 \mu \wedge 2+8(\lambda+\mu) c_{1}\right.\right. \\
& \left.\left.+5 \lambda c_{4}+6 \mu c_{4}\right) e_{2}-4 c_{3}\left((\lambda+\mu) e_{1}-\mu e_{2}+2 \lambda e_{3}+2 \mu e_{3}\right)\right) \text {, } \\
& \chi_{31}=1 / 4\left(-4 \lambda-4 \mu-(3 \lambda \mu) /(\lambda+\mu)-4 c_{1}-6 c_{3}-4 c_{4}-\left(3 \lambda c_{4}\right) /(2(\lambda+\mu))\right), \\
& \chi_{32}=-1 /(8(\lambda+\mu))\left(8 \lambda \wedge 2+10 \lambda \mu+4 \mu \wedge 2+16(\lambda+\mu) c_{1}+4(4 \lambda+5 \mu) c_{3}+7 \lambda c_{4}+10 \mu c_{4}\right) \text {, } \\
& \chi_{33}=1 /\left(8(\lambda+\mu) e_{2}\right)\left(\left(6 \lambda \mu+4 \mu \wedge 2-8(\lambda+\mu) c_{1}\right.\right. \\
& \left.\left.+\lambda c_{4}-2 \mu c_{4}\right) e_{2}+4 c_{3}\left((\lambda+\mu) e_{1}-\mu e_{2}+2 \lambda e_{3}+2 \mu e_{3}\right)\right) \\
& \chi_{34}=1 /\left(8(\lambda+\mu) c_{3}\right)\left(\left(6 \lambda \mu+4 \mu \wedge 2-8(\lambda \mu) c_{1}\right.\right. \\
& \left.\left.+\lambda c_{4}-2 \mu c_{4}\right) e_{2}+4 c_{3}\left((\lambda+\mu) e_{1}-\mu e_{2}+2 \lambda e_{3}+2 \mu e_{3}\right)\right) \text {, } \\
& \chi_{35}=\left(2 \lambda \mu+2 \mu \wedge 2+4(\lambda+\mu) c_{1}+2(\lambda+2 \mu) c_{3}+2 \lambda c_{4}+3 \mu c_{4}\right) /(4(\lambda+\mu)) \text {, } \\
& \chi_{36}=1 /\left(8(\lambda+\mu) e_{2}\right)\left(\left(6 \lambda \mu+4 \mu \wedge 2+8(\lambda+\mu) c_{1}\right.\right. \\
& \left.\left.+5 \lambda c_{4}+6 \mu c_{4}\right) e_{2}-4 c_{3}\left((\lambda+\mu) e_{1}-\mu e_{2}+2 \lambda e_{3}+2 \mu e_{3}\right)\right) \text {, } \\
& \chi_{37}=1 /\left(8(\lambda+\mu) c_{3}\right)\left(\left(6 \lambda \mu+4 \mu \wedge 2+8(\lambda+\mu) c_{1}\right.\right. \\
& \left.\left.+5 \lambda c_{4}+6 \mu c_{4}\right) e_{2}-4 c_{3}\left((\lambda+\mu) e_{1}-\mu e_{2}+2 \lambda e_{3}+2 \mu e_{3}\right)\right) \text {, } \\
& \chi_{38}=e_{1}+2\left(e_{2}+e_{3}\right) \text {, } \\
& \chi_{39}=1 /\left(4 e_{2}^{2}(\lambda+\mu) \wedge 2\right)\left(12 c_{3}^{2}(\lambda+\mu) \wedge 2\left(\mu_{1}+\mu_{2}\right)\right. \\
& -4 c_{3} e_{2}(\lambda+\mu)\left(\lambda\left(2+3 v_{11}+2 v_{12}+2 v_{2}+2 v_{7}\right)+\mu\left(1+3 v_{11}+2 v_{12}+2 v_{2}+2 v_{4}\right.\right. \\
& \left.\left.+2 v_{7}+2 v_{8}\right)\right)+e_{2}^{2}\left(3 e_{2} \lambda \wedge 2+4 e_{3} \lambda \wedge 2+4 e_{2} \lambda \mu+8 e_{3} \lambda \mu+2 e_{2} \mu \wedge 2\right. \\
& +4 e_{3} \mu \wedge 2+2 e_{1}(\lambda+\mu) \wedge 2+4 \lambda \wedge 2 v_{1}+8 \lambda v v_{1}+4 \mu \wedge 2 v_{1}+4 \lambda \mu v_{10}+4 \mu \wedge 2 v_{10} \\
& +8 \lambda \wedge 2 v_{13}+16 \lambda \mu v_{13}+8 \mu \wedge 2 v_{13}+4 \lambda \mu v_{14}+4 \mu \wedge 2 v_{14}+4 \mu \wedge 2 v_{3}+4 \lambda \wedge 2 v_{5} \\
& \left.\left.+8 \lambda \mu v_{5}+4 \mu \wedge 2 v_{5}+6 \lambda \wedge 2 v_{6}+8 \lambda \mu v_{6}+4 \mu \wedge 2 v_{6}+4 \lambda \wedge 2 v_{9}+8 \lambda x \mu v_{9}+4 \mu \wedge 2 v_{9}\right)\right) \text {, } \\
& \chi_{40}=1 /\left(4 c_{3}^{2}(\lambda+\mu) \wedge 2\right)\left(12 c_{3}^{2}(\lambda+\mu) \wedge 2\left(\mu_{1}+\mu_{2}\right)\right. \\
& -4 c_{3} e_{2}(\lambda+\mu)\left(\lambda\left(2+3 v_{11}+2 v_{12}+2 v_{2}+2 v_{7}\right)+\mu\left(1+3 v_{11}+2 v_{12}+2 v_{2}+2 v_{4}\right.\right. \\
& \left.\left.+2 v_{7}+2 v_{8}\right)\right)+e_{2}^{2}\left(3 e_{2} \lambda \wedge 2+4 e_{3} \lambda \wedge 2+4 e_{2} \lambda \mu+8 e_{3} \lambda \mu+2 e_{2} \mu \wedge 2\right. \\
& +4 e_{3} \mu \wedge 2+2 e_{1}(\lambda+\mu) \wedge 2+4 \lambda \wedge 2 v_{1}+8 \lambda v v_{1}+4 \mu \wedge 2 v_{1}+4 \lambda \mu v_{10}+4 \mu \wedge 2 v_{10} \\
& +8 \lambda \wedge 2 v_{13}+16 \lambda \mu v_{13}+8 \mu \wedge 2 v_{13}+4 \lambda \mu v_{14}+4 \mu \wedge 2 v_{14}+4 \mu \wedge 2 v_{3}+4 \lambda \wedge 2 v_{5} \\
& \left.\left.+8 \lambda \mu v_{5}+4 \mu \wedge 2 v_{5}+6 \lambda \wedge 2 v_{6}+8 \lambda \mu v_{6}+4 \mu \wedge 2 v_{6}+4 \lambda \wedge 2 v_{9}+8 \lambda \mu v_{9}+4 \mu \wedge 2 v_{9}\right)\right) \text {, } \\
& \chi_{41}=1 /\left(2 c_{3} e_{2}(\lambda+\mu) \wedge 2\right)\left(12 c_{3}^{2}(\lambda+\mu) \wedge 2\left(\mu_{1}+\mu_{2}\right)\right. \\
& -4 c_{3} e_{2}(\lambda+\mu)\left(\lambda\left(2+3 v_{11}+2 v_{12}+2 v_{2}+2 v_{7}\right)+\mu\left(1+3 v_{11}+2 v_{12}+2 v_{2}+2 v_{4}\right.\right. \\
& \left.\left.\left.+2 v_{7}+2 v_{8}\right)\right)+4 \mu \wedge 2 \lambda_{8}+3 \lambda \wedge 2 \lambda_{9}\right)+e_{2}^{2}\left(3 e_{2} \lambda \wedge 2+4 e_{3} \lambda \wedge 2+4 e_{2} \lambda \mu+8 e_{3} \lambda \mu\right. \\
& +2 e_{2} \mu \wedge 2+4 e_{3} \mu \wedge 2+2 e_{1}(\lambda+\mu) \wedge 2+4 \lambda \wedge 2 v_{1}+8 \lambda v v_{1}+4 \mu \wedge 2 v_{1}+4 \lambda \mu v_{10} \\
& +4 \mu \wedge 2 v_{10}+8 \lambda \wedge 2 v_{13}+16 \lambda \mu v_{13}+8 \mu \wedge 2 v_{13}+4 \lambda \mu v_{14}+4 \mu \wedge 2 v_{14}+4 \mu \wedge 2 v_{3}+4 \lambda \wedge 2 v_{5} \\
& \left.\left.+8 \lambda \mu v_{5}+4 \mu \wedge 2 v_{5}+6 \lambda \wedge 2 v_{6}+8 \lambda \mu v_{6}+4 \mu \wedge 2 v_{6}+4 \lambda \wedge 2 v_{9}+8 \lambda \mu v_{9}+4 \mu \wedge 2 v_{9}\right)\right) \text {, } \\
& \chi_{42}=\left(e_{2} \lambda \wedge 2\right) /(4(\lambda+\mu) \wedge 2) \text {, }
\end{aligned}
$$




$$
\begin{aligned}
& \chi_{43}=e_{1} / 2+e_{3}+1 / 4 e_{2}(2+\lambda \wedge 2 /(\lambda+\mu) \wedge 2)+v_{1}+v_{10}-\left(\lambda v_{10}\right) /(\lambda+\mu)+2 v_{13}+v_{14} \\
& -\left(\lambda v_{14}\right) /(\lambda+\mu)+v_{3}+(\lambda \wedge 2 v 3) /(\lambda+\mu) \wedge 2-\left(2 \lambda v_{3}\right) /(\lambda+\mu)+v_{5}+v_{6} \\
& +\left(\lambda \wedge 2 v_{6}\right) /(2(\lambda+\mu) \wedge 2)+v_{9} \\
& \chi_{44}=-2 c_{1}-3 c_{3}-2\left(c_{4}+\lambda+\mu\right) \text {, } \\
& \chi_{45}=1 /\left(4 e_{2}(\lambda+\mu)\right)\left(e _ { 2 } \left(5 c_{4} \lambda+6 c_{4} \mu\right.\right. \\
& \left.\left.+6 \lambda \mu+4 \mu \wedge 2+8 c_{1}(\lambda+\mu)\right)-4 c_{3}\left(2 e_{3} \lambda-e_{2} \mu+2 e_{3} \mu+e_{1}(\lambda+\mu)\right)\right), \\
& \chi_{46}=1 /\left(4 c_{3}(\lambda+\mu)\right)\left(e _ { 2 } \left(5 c_{4} \lambda+6 c_{4} \mu\right.\right. \\
& \left.\left.+6 \lambda \mu+4 \mu \wedge 2+8 c_{1}(\lambda+\mu)\right)-4 c_{3}\left(2 e_{3} \lambda-e_{2} \mu+2 e_{3} \mu+e_{1}(\lambda+\mu)\right)\right), \\
& \chi_{47}=1 /\left(4 e_{2}^{2}(\lambda+\mu) \wedge 2\right)\left(-e_{2}^{2}\left(12 c_{4} \lambda \wedge 2+3 \lambda \wedge 3+12 \lambda \wedge 2 \lambda_{1}+4 \lambda \wedge 2 \lambda_{3}\right.\right. \\
& +12 \lambda \wedge 2 \lambda_{5}+4 \lambda \wedge 2 \lambda_{6}+14 \lambda \wedge 2 \lambda_{7}+6 \lambda \wedge 2 \lambda_{8}+12 \lambda \wedge 2 \lambda_{9}+24 c_{4} \lambda \mu+20 \lambda \wedge 2 \mu \\
& +24 \lambda \lambda_{1} \mu+16 \lambda \lambda_{3} \mu+8 \lambda \lambda_{4} \mu+24 \lambda_{5} \mu+16 \lambda_{6} \lambda_{6} \mu+24 \lambda \lambda_{7} \mu+16 \lambda_{8} \mu+24 \lambda \lambda_{9} \mu \\
& +12 c_{4} \mu \wedge 2+30 \lambda_{\mu} \wedge 2+12 \lambda_{1} \mu \wedge 2+12 \lambda_{2} \mu \wedge 2+12 \lambda_{3} \mu \wedge 2+12 \lambda_{4} \mu \wedge 2+12 \lambda_{5} \mu \wedge 2 \\
& \left.+12 \lambda_{6} \mu \wedge 2+12 \lambda_{7} \mu \wedge 2+12 \lambda_{8} \mu \wedge 2+12 \lambda_{9} \mu \wedge 2+12 \mu \wedge 3+12 c_{1}(\lambda+\mu) \wedge 2\right) \\
& +2 c_{3}^{2}(\lambda+\mu) \wedge\left(-1+4 e_{1}+4 e_{2}-4 v_{11}-2 v_{12}-2 v_{2}-2 v_{4}-2 v_{7}-2 v_{8}\right) \\
& +c_{3} e_{2}\left(-e_{2}(5 \lambda \wedge 2+16 \lambda \mu+12 \mu \wedge 2)+4(\lambda+\mu)\left(2 \lambda v_{1}+2 \mu v_{1}+2 \lambda v_{10}+4 \mu v_{10}+4 \lambda v_{13}\right.\right. \\
& \left.\left.\left.+4 \mu v_{13}+\lambda v_{14}+2 \mu v_{14}+2 \mu v_{3}+2 \lambda v_{5}+2 \mu v_{5}+2 \lambda v_{6}+2 \mu v_{6}+4 \lambda v_{9}+4 \mu v_{9}\right)\right)\right) \text {, } \\
& \chi_{48}=1 /\left(4 c_{3}^{2}(\lambda+\mu) \wedge 2\right)\left(-e_{2}^{2}\left(12 c_{4} \lambda \wedge 2+3 \lambda \wedge 3+12 \lambda \wedge 2 \lambda_{1}+4 \lambda \wedge 2 \lambda_{3}\right.\right. \\
& +12 \lambda \wedge 2 \lambda_{5}+4 \lambda \wedge 2 \lambda_{6}+14 \lambda \wedge 2 \lambda_{7}+6 \lambda \wedge 2 \lambda_{8}+12 \lambda \wedge 2 \lambda_{9}+24 c_{4} \lambda \mu+20 \lambda \wedge 2 \mu \\
& +24 \lambda \lambda_{1} \mu+16 \lambda \lambda_{3} \mu+8 \lambda \lambda_{4} \mu+24 \lambda_{5} \mu+16 \lambda_{6} \mu+24 \lambda \lambda_{7} \mu+16 \lambda_{8} \mu+24 \lambda \lambda_{9} \mu \\
& +12 c_{4} \mu \wedge 2+30 \lambda_{\mu} \wedge 2+12 \lambda_{1} \mu \wedge 2+12 \lambda_{2} \mu \wedge 2+12 \lambda_{3} \mu \wedge 2+12 \lambda_{4} \mu \wedge 2+12 \lambda_{5} \mu \wedge 2 \\
& \left.+12 \lambda_{6} \mu \wedge 2+12 \lambda_{7} \mu \wedge 2+12 \lambda_{8} \mu \wedge 2+12 \lambda_{9} \mu \wedge 2+12 \mu \wedge 3+12 c_{1}(\lambda+\mu) \wedge 2\right) \\
& +2 c_{3}^{2}(\lambda+\mu) \wedge\left(-1+4 e_{1}+4 e_{2}-4 v_{11}-2 v_{12}-2 v_{2}-2 v_{4}-2 v_{7}-2 v_{8}\right) \\
& +c_{3} e_{2}\left(-e_{2}(5 \lambda \wedge 2+16 \lambda \mu+12 \mu \wedge 2)+4(\lambda+\mu)\left(2 \lambda v_{1}+2 \mu v_{1}+2 \lambda v_{10}+4 \mu v_{10}+4 \lambda v_{13}\right.\right. \\
& \left.\left.\left.+4 \mu v_{13}+\lambda v_{14}+2 \mu v_{14}+2 \mu v_{3}+2 \lambda v_{5}+2 \mu v_{5}+2 \lambda v_{6}+2 \mu v_{6}+4 \lambda v_{9}+4 \mu v_{9}\right)\right)\right) \text {, } \\
& \chi_{49}=1 /\left(2 c_{3} e_{2}(\lambda+\mu) \wedge 2\right)\left(-e_{2}^{2}\left(12 c_{4} \lambda \wedge 2+3 \lambda \wedge 3+12 \lambda \wedge 2 \lambda_{1}+4 \lambda \wedge 2 \lambda_{3}\right.\right. \\
& +12 \lambda \wedge 2 \lambda_{5}+4 \lambda \wedge 2 \lambda_{6}+14 \lambda \wedge 2 \lambda_{7}+6 \lambda \wedge 2 \lambda_{8}+12 \lambda \wedge 2 \lambda_{9}+24 c_{4} \lambda \mu+20 \lambda \wedge 2 \mu \\
& +24 \lambda \lambda_{1} \mu+16 \lambda \lambda_{3} \mu+8 \lambda \lambda_{4} \mu+24 \lambda_{5} \mu+16 \lambda_{6} \mu+24 \lambda \lambda_{7} \mu+16 \lambda_{8} \mu+24 \lambda \lambda_{9} \mu \\
& +12 c_{4} \mu \wedge 2+30 \lambda \mu \wedge 2+12 \lambda_{1} \mu \wedge 2+12 \lambda_{2} \mu \wedge 2+12 \lambda_{3} \mu \wedge 2+12 \lambda_{4} \mu \wedge 2+12 \lambda_{5} \mu \wedge 2 \\
& \left.+12 \lambda_{6} \mu \wedge 2+12 \lambda_{7} \mu \wedge 2+12 \lambda_{8} \mu \wedge 2+12 \lambda_{9} \mu \wedge 2+12 \mu \wedge 3+12 c_{1}(\lambda+\mu) \wedge 2\right) \\
& +2 c_{3}^{2}(\lambda+\mu) \wedge\left(-1+4 e_{1}+4 e_{2}-4 v_{11}-2 v_{12}-2 v_{2}-2 v_{4}-2 v_{7}-2 v_{8}\right) \\
& +c_{3} e_{2}\left(-e_{2}(5 \lambda \wedge 2+16 \lambda \mu+12 \mu \wedge 2)+4(\lambda+\mu)\left(2 \lambda v_{1}+2 \mu v_{1}+2 \lambda v_{10}+4 \mu v_{10}+4 \lambda v_{13}\right.\right. \\
& \left.\left.\left.+4 \mu v_{13}+\lambda v_{14}+2 \mu v_{14}+2 \mu v_{3}+2 \lambda v_{5}+2 \mu v_{5}+2 \lambda v_{6}+2 \mu v_{6}+4 \lambda v_{9}+4 \mu v_{9}\right)\right)\right) \text {, } \\
& \chi_{50}=-\left(\lambda \wedge 2\left(c_{3}+\lambda\right)\right) /(4(\lambda+\mu) \wedge 2)
\end{aligned}
$$




$$
\begin{aligned}
& \chi_{51}=-1 /(4(\lambda+\mu) \wedge 2)\left(12 c_{4} \lambda \wedge 2+3 \lambda \wedge 3+12 \lambda \wedge 2 \lambda_{1}+4 \lambda \wedge 2 \lambda_{3}+12 \lambda \wedge 2 \lambda_{5}\right. \\
& +4 \lambda \wedge 2 \lambda_{6}+14 \lambda \wedge 2 \lambda_{7}+6 \lambda \wedge 2 \lambda_{8}+12 \lambda \wedge 2 \lambda_{9}+24 c_{4} \lambda \mu+20 \lambda \wedge 2 \mu+24 \lambda \lambda_{1} \mu+16 \lambda \lambda_{3} \mu \\
& +8 \lambda \lambda_{4} \mu+24 \lambda \lambda_{5} \mu+16 \lambda \lambda_{6} \mu+24 \lambda \lambda_{7} \mu+16 \lambda_{8}+24 \lambda \lambda_{9} \mu+12 c_{4} \mu \wedge 2+30 \lambda \mu \wedge 2 \\
& +12 \lambda_{1} \mu \wedge 2+12 \lambda_{2} \mu \wedge 2+12 \lambda_{3} \mu \wedge 2+12 \lambda_{4} \mu \wedge 2+12 \lambda_{5} \mu \wedge 2+12 \lambda_{6} \mu \wedge 2+12 \lambda_{7} \mu \wedge 2 \\
& \left.+12 \lambda_{8} \mu \wedge 2+12 \lambda_{9} \mu \wedge 2+12 \mu \wedge 3+12 c_{1}(\lambda+\mu) \wedge 2+c_{3}(9 \lambda \wedge 2+20 \lambda \mu+12 \mu \wedge 2)\right) \text {, } \\
& \chi_{52}=1 /\left(4 e_{2}(\lambda+\mu)\right)\left(-e_{2}\left(5 c_{4} \lambda+6 c_{4} \mu\right.\right. \\
& \left.+6 \lambda \mu+4 \mu \wedge 2+8 c_{1}(\lambda+\mu)+4 c_{3}\left(2 e_{3} \lambda-e_{2} \mu+2 e_{3} \mu+e_{1}(\lambda+\mu)\right)\right), \\
& \chi_{53}=1 /\left(4 c_{3}(\lambda+\mu)\right)\left(-e_{2}\left(5 c_{4} \lambda+6 c_{4} \mu+6 \lambda \mu\right.\right. \\
& \left.+4 \mu \wedge 2+8 c_{1}(\lambda+\mu)+4 c_{3}\left(2 e_{3} \lambda-e_{2} \mu+2 e_{3} \mu+e_{1}(\lambda+\mu)\right)\right) \text {, } \\
& \chi_{54}=\left(2 c_{4} \lambda+3 c_{4} \mu+2 \lambda \mu+2 \mu \wedge 2+4 c_{1}(\lambda+\mu)+2 c_{3}(\lambda+2 \mu)\right) /(4(\lambda+\mu)) \text {, } \\
& \chi_{55}=-1 /\left(4 e_{2}(\lambda+\mu) \wedge 2\right)\left(12 c_{1} e_{2}(\lambda+\mu) \wedge 2\right. \\
& +e_{2}\left(12 c_{4} \lambda \wedge 2+3 \lambda \wedge 3+12 \lambda \wedge 2 \lambda_{1}+4 \lambda \wedge 2 \lambda_{3}+12 \lambda \wedge 2 \lambda_{5}+4 \lambda \wedge 2 \lambda_{6}+14 \lambda \wedge 2 \lambda_{7}\right. \\
& +6 \lambda \wedge 2 \lambda_{8}+12 \lambda \wedge 2 \lambda_{9}+24 c_{4} \lambda \mu+20 \lambda \wedge 2 \mu+24 \lambda \lambda_{1} \mu+16 \lambda \lambda_{3} \mu+8 \lambda \lambda_{4}+24 \lambda \lambda_{5} \mu \\
& +16 \lambda_{6} \mu+24 \lambda \lambda_{7} \mu+16 \lambda_{8} \mu+24 \lambda \lambda_{9} \mu+12 c_{4} \mu \wedge 2+30 \lambda \mu \wedge 2+12 \lambda_{1} \mu \wedge 2 \\
& +12 \lambda_{2} \mu \wedge 2+12 \lambda_{3} \mu \wedge 2+12 \lambda_{4} \mu \wedge 2+12 \lambda_{5} \mu \wedge 2+12 \lambda_{6} \mu \wedge 2+12 \lambda_{7} \mu \wedge 2 \\
& \left.+12 \lambda_{8} \mu \wedge 2+12 \lambda_{9} \mu \wedge 2+12 \mu \wedge 3\right)+c_{3}\left(7 e_{2} \lambda \wedge 2+18 e_{2} \lambda \mu+12 e_{2} \mu \wedge 2-4 \lambda \wedge 2 v_{1}\right. \\
& -8 \lambda \mu v_{1}-4 \mu \wedge 2 v_{1}-4 \lambda \wedge 2 v_{10}-12 \lambda \mu v_{10}-8 \mu \wedge 2 v_{10}-8 \lambda \wedge 2 v_{13}-16 \lambda \mu v_{13} \\
& -8 \mu \wedge 2 v_{13}-2 \mu \wedge 2 v_{14}-6 \lambda \mu v_{14}-4 \mu \wedge 2 v_{14}-4 \lambda \mu v_{3}-4 \mu \wedge 2 v_{3}-4 \lambda \wedge 2 v_{5} \\
& \left.\left.-8 \lambda \mu v_{5}-4 \mu \wedge 2 v_{5}-4 \lambda \wedge 2 v_{6}-8 \lambda \mu v_{6}-4 \mu \wedge 2 v_{6}-8 \lambda \wedge 2 v_{9}-16 \lambda \mu v_{9}-8 \mu \wedge 2 v_{9}\right)\right) \text {, } \\
& \chi_{56}=-1 /\left(4 c_{3}(\lambda+\mu) \wedge 2\right)\left(12 c_{1} e_{2}(\lambda+\mu) \wedge 2\right. \\
& +e_{2}\left(12 c_{4} \lambda \wedge 2+3 \lambda \wedge 3+12 \lambda \wedge 2 \lambda_{1}+4 \lambda \wedge 2 \lambda_{3}+12 \lambda \wedge 2 \lambda_{5}+4 \lambda \wedge 2 \lambda_{6}+14 \lambda \wedge 2 \lambda_{7}\right. \\
& +6 \lambda \wedge 2 \lambda_{8}+12 \lambda \wedge 2 \lambda_{9}+24 c_{4} \lambda \mu+20 \lambda \wedge 2 \mu+24 \lambda \lambda_{1} \mu+16 \lambda \lambda_{3} \mu+8 \lambda \lambda_{4}+24 \lambda \lambda_{5} \mu \\
& +16 \lambda_{6} \mu+24 \lambda \lambda_{7} \mu+16 \lambda \lambda_{8} \mu+24 \lambda \lambda_{9} \mu+12 c_{4} \mu \wedge 2+30 \lambda \mu \wedge 2+12 \lambda_{1} \mu \wedge 22 \\
& +12 \lambda_{2} \mu \wedge 2+12 \lambda_{3} \mu \wedge+12 \lambda_{4} \mu \wedge 2+12 \lambda_{5} \mu \wedge 2+12 \lambda_{6} \mu \wedge 2+12 \lambda_{7} \mu \wedge 2+12 \lambda_{8} \mu \wedge 2 \\
& \left.+12 \lambda_{9} \mu \wedge 2+12 \mu \wedge 3\right)+c_{3}\left(7 e_{2} \lambda \wedge 2+18 e_{2} \lambda \mu+12 e_{2} \mu \wedge 2-4 \lambda \wedge 2 v_{1}\right. \\
& -8 \lambda \mu v_{1}-4 \mu \wedge 2 v_{1}-4 \lambda \wedge 2 v_{10}-12 \lambda \mu v_{10}-8 \mu \wedge 2 v_{10}-8 \lambda \wedge 2 v_{13}-16 \lambda \mu v_{13} \\
& -8 \mu \wedge 2 v_{13}-2 \mu \wedge 2 v_{14}-6 \lambda \mu v_{14}-4 \mu \wedge 2 v_{14}-4 \lambda \mu v_{3}-4 \mu \wedge 2 v_{3}-4 \lambda \wedge 2 v_{5} \\
& \left.\left.-8 \lambda \mu v_{5}-4 \mu \wedge 2 v_{5}-4 \lambda \wedge 2 v_{6}-8 \lambda \mu v_{6}-4 \mu \wedge 2 v_{6}-8 \lambda \wedge 2 v_{9}-16 \lambda \mu v_{9}-8 \mu \wedge 2 v_{9}\right)\right) \text {, } \\
& \xi_{1}=-\frac{c_{3}+\lambda}{2(\lambda+\mu)} \\
& \xi_{2}=-\frac{c_{3}+3 \lambda+2 \mu}{2(\lambda+\mu)}, \\
& \xi_{3}=\left(\left(2-4 \epsilon_{1}-4 \epsilon_{2}\right) c_{3}^{2}+c_{4}\left(-e_{1} e_{2}-5 e_{2}^{2}+2 e_{2} e_{3}-5 \lambda+10 \epsilon_{1} \lambda+10 \epsilon_{2} \lambda-4 \mu+8 \epsilon_{1} \mu+8 \epsilon_{2} \mu\right)\right.
\end{aligned}
$$




$$
\begin{aligned}
& -2\left(2 c_{1} e_{2}^{2}-2 c_{1} e_{2} e_{3}+2 c_{1} \lambda-4 \epsilon_{1} c_{1} \lambda-4 \epsilon_{2} c_{1} \lambda\right. \\
& +e_{1}^{2} \lambda+5 e_{1} e_{3} \lambda+6 e_{3}^{2} \lambda+2 c_{1} \mu-4 \epsilon_{1} c_{1} \mu-4 \epsilon_{2} c_{1} \mu+e_{1}^{2} \mu+3 e_{1} e_{2} \mu+4 e_{2}^{2} \mu \\
& \left.+4 e_{1} e_{3} \mu+4 e_{2} e_{3} \mu+4 e_{3}^{2} \mu+4 \lambda \mu-8 \epsilon_{1} \mu-8 \epsilon_{2} \lambda \mu+2 \mu^{2}-4 \epsilon_{1} \mu^{2}-4 \epsilon_{2} \mu^{2}\right) \\
& +c_{3}\left(\left(-1+2 \epsilon_{1}+2 \epsilon_{2}\right) c_{4}+2\left(2 e_{1} e_{2}+e_{2}^{2}-e_{1} e_{3}+4 e_{2} e_{3}-2 e_{3}^{2}+\lambda-2 \epsilon_{1} \lambda-2 \epsilon_{2} \lambda\right.\right. \\
& \left.\left.\left.-2 \mu+4 \epsilon_{1} \mu+4 \epsilon_{2} \mu\right)\right)\right) /\left(\left(-2+4 \epsilon_{1}+4 \epsilon_{2}\right) c_{3}^{2}+c_{3}\left(c_{4}-2 \epsilon_{1} c_{4}-2 \epsilon_{2} c_{4}-4 e_{1} e_{2}+2 e_{1} e_{3}\right.\right. \\
& \left.-10 e_{2} e_{3}+4 e_{3}^{2}+6 \mu-12 \epsilon_{1} \mu-12 \epsilon_{2} \mu\right)+c_{4}\left(e_{1} e_{2}+5 e_{2}^{2}-2 e_{2} e_{3}+5 \lambda-10 \epsilon_{1} \lambda-10 \epsilon_{2} \lambda\right. \\
& \left.+4 \mu-8 \epsilon_{1} \mu-8 \epsilon_{2} \mu\right)+2\left(c_{1} e_{2}^{2}-2 c_{1} e_{2} e_{3}+2 c_{1} \lambda-4 \epsilon_{1} c_{1} \lambda-4 \epsilon_{2} c_{1} \lambda+e_{1}^{2} \lambda+5 e_{1} e_{3} \lambda\right. \\
& +6 e_{3}^{2}+2 c_{1} \mu-4 \epsilon_{1} c_{1} \mu-4 \epsilon_{2} c_{1} \mu+e_{1}^{2} \mu+3 e_{1} e_{2} \mu+4 e_{2}^{2} \mu+4 e_{1} e_{3} \mu+4 e_{2} e_{3} \mu+4 e_{3}^{2} \mu \\
& \left.\left.+4 \lambda \mu-8 \epsilon_{1} \lambda \mu-8 \epsilon_{2} \lambda \mu+2 \mu^{2}-4 \epsilon_{1} \mu^{2}-4 \epsilon_{2} \mu^{2}\right)\right) \text {, } \\
& \xi_{4}=\left(2 c_{3}\left(c_{3}\left(e_{2}-e_{3}\right)-3 e_{3} \lambda-e_{2} \mu-2 e_{3} \mu-e_{1}(\lambda+\mu)\right)\right) /\left(\left(-2+\epsilon_{1}+4 \epsilon_{2}\right) c_{3}^{2}+c_{3}\left(c_{4}\right.\right. \\
& \left.-2 \epsilon_{1} c_{4}-2 \epsilon_{2} c_{4}-4 e_{1} e_{2}+2 e_{1} e_{3}-10 e_{2} e_{3}+4 e_{3}^{2}+6 \mu-12 \epsilon_{1} \mu-12 \epsilon_{2} \mu\right)+c_{4}\left(e_{1} e_{2}\right. \\
& \left.+5 e_{2}^{2}-2 e_{2} e_{3}+5 \lambda-10 \epsilon_{1} \lambda-10 \epsilon_{2} \lambda+4 \mu-8 \epsilon_{1} \mu-8 \epsilon_{2} \mu\right)+2\left(2 c_{1} e_{2}^{2}-2 c_{1} e_{2} e_{3}+2 c_{1} \lambda\right. \\
& -4 \epsilon_{1} c_{1} \lambda-4 \epsilon_{2} c_{1} \lambda+e_{1}^{2} \lambda+5 e_{1} e_{3} \lambda+6 e_{3}^{2} \lambda+2 c_{1} \mu-4 \epsilon_{1} c_{1} \mu-4 \epsilon_{2} c_{1} \mu+e_{1}^{2} \mu+3 e_{1} e_{2} \mu \\
& \left.\left.+4 e_{2}^{2} \mu+4 e_{1} e_{3} \mu+4 e_{2} e_{3} \mu+4 e_{3}^{2} \mu+4 \lambda \mu-8 \epsilon_{1} \lambda \mu-8 \epsilon_{2} \lambda \mu+2 \mu^{2}-4 \epsilon_{1} \mu^{2}-4 \epsilon_{2} \mu^{2}\right)\right) \text {, } \\
& \xi_{5}=\left(\left(2-4 \epsilon_{1}-4 \epsilon_{2}\right) c_{3}^{3}+c_{3}^{2}\left(\left(-1+2 \epsilon_{1}+2 \epsilon_{2}\right) c_{4}\right.\right. \\
& \left.+2\left(2 e_{1} e_{2}-e_{1} e_{3}+5 e_{2} e_{3}-2 e_{3}^{2}+2 \lambda-4 \epsilon_{1} \lambda-4 \epsilon_{2} \lambda-2 \mu+4 \epsilon_{1} \mu+4 \epsilon_{2} \mu\right)\right) \\
& -c_{3}\left(c_{4}\left(e_{1} e_{2}+5 e_{2}^{2}-2 e_{2} e_{3}+6 \lambda-12 \epsilon_{1} \lambda-12 \epsilon_{2} \lambda+4 \mu-8 \epsilon_{1} \mu-8 \epsilon_{2} \mu\right)\right. \\
& +2\left(2 c_{1} e_{2}^{2}-2 c_{1} e_{2} e_{3}+2 c_{1} \lambda-4 \epsilon_{1} c_{1} \lambda-4 \epsilon_{2} c_{1} \lambda+e_{1}^{2} \lambda-3 e_{1} e_{2} \lambda-e_{2}^{2} \lambda+6 e_{1} e_{3} \lambda-7 e_{2} e_{3} \lambda\right. \\
& +8 e_{3}^{2} \lambda-\lambda^{2}+2 \epsilon_{2} \lambda^{2}+2 c_{1} \mu-4 \epsilon_{1} c_{1} \mu-4 \epsilon_{2} c_{1} \mu+e_{1}^{2} \mu+2 e_{1} e_{2} \mu+3 e_{2}^{2} \mu \\
& \left.\left.+4 e_{1} e_{3} \mu+2 e_{2} e_{3} \mu+4 e_{3}^{2} \mu+6 \lambda \mu-12 \epsilon_{1} \lambda \mu-12 \epsilon_{2} \lambda \mu+2 \mu^{2}-4 \epsilon_{1} \mu^{2}-4 \epsilon_{2} \mu^{2}\right)\right) \\
& +\lambda\left(c_{4}\left(-e_{1} e_{2}-5 e_{2}^{2}+2 e_{2} e_{3}-5 \lambda+10 \epsilon_{1} \lambda+10 \epsilon_{2} \lambda-4 \mu+8 \epsilon_{1} \mu+8 \epsilon_{2} \mu\right)\right. \\
& -2\left(2 c_{1} e_{2}^{2}-2 c_{1} e_{2} e_{3}+2 c_{1} \lambda-4 \epsilon_{1} c_{1} \lambda-4 \epsilon_{2} c_{1} \lambda\right. \\
& +e_{1}^{2} \lambda+5 e_{1} e_{3} \mu+6 e_{3}^{2} \lambda+2 c_{1} \mu-4 \epsilon_{1} c_{1} \mu-4 \epsilon_{2} c_{1} \mu+e_{1}^{2} \mu+3 e_{1} e_{2} \mu+4 e_{2}^{2} \mu \\
& \left.\left.\left.+4 e_{1} e_{3} \mu+4 e_{2} e_{3} \mu+4 e_{3}^{2} \mu+4 \lambda \mu-8 \epsilon_{1} \lambda \mu-\epsilon_{2} \lambda \mu+2 \mu^{2}-4 \epsilon_{1} \mu^{2}-4 \epsilon_{2} \mu^{2}\right)\right)\right) / \\
& \left(2 ( \lambda + \mu ) \left(\left(2-4 \epsilon_{1}-4 \epsilon_{2}\right) c_{3}^{2}+c_{4}\left(-e_{1} e_{2}-5 e_{2}^{2}+2 e_{2} e_{3}\right.\right.\right. \\
& \left.-5 \lambda+10 \epsilon_{1} \lambda+10 \epsilon_{2} \lambda-4 \mu+8 \epsilon_{1} \mu+8 \epsilon_{2} \mu\right)-2\left(2 c_{1} e_{2}^{2}-2 c_{1} e_{2} e_{3}+2 c_{1} \lambda\right. \\
& -4 \epsilon_{1} c_{1} \lambda-4 \epsilon_{2} c_{1} \lambda+e_{1}^{2} \lambda+5 e_{1} e_{3} \lambda+6 e_{3}^{2} \lambda+2 c_{1} \mu-4 \epsilon_{1} c_{1} \mu+e_{1}^{2} \mu+3 e_{1} e_{2} \mu \\
& +4 e_{2}^{2} \mu+4 e_{1} e_{3} \mu+4 e_{2} e_{3} \mu+4 \lambda \mu-8 \epsilon_{1} \lambda \mu-8 \epsilon_{2} \lambda \mu+2 \mu^{2}-4 \epsilon_{1} \mu^{2}-4 \epsilon_{2} \mu^{2} \\
& \left.\left.+c_{3}\left(\left(-1+2 \epsilon_{1}+2 \epsilon_{2}\right) c_{4}+2\left(2 e_{1} e_{2}-e_{1} e_{3}+5 e_{2} e_{3}-2 e_{3}^{2}-3 \mu+6 \epsilon_{1} \mu+6 \epsilon_{2} \mu\right)\right)\right)\right) \text {, }
\end{aligned}
$$




$$
\begin{aligned}
& \xi_{6}=-\left(4 c _ { 3 } \left(\left(-1+2 \epsilon_{1}+2 \epsilon_{2}\right) c_{3}^{2}+2 c_{4} e_{3}^{2}-2 c_{4} e_{2} e_{3}\right.\right. \\
& +2 c_{4} \lambda-4 \epsilon_{1} c_{4} \lambda-4 \epsilon_{2} c_{4} \lambda+e_{1}^{2} \lambda+5 e_{1} e_{3} \lambda+6 e_{3}^{2} \lambda+2 c_{4} \mu-4 \epsilon_{1} c_{4} \mu-4 \epsilon_{2} c_{4} \mu \\
& +e_{1}^{2} \mu+2 e_{1} e_{2} \mu+3 e_{2}^{2} \mu+4 e_{1} e_{3} \mu+2 e_{2} e_{3} \mu+4 e_{3}^{2} \mu+3 \lambda \mu-6 \epsilon_{1} \lambda \mu-6 \epsilon_{2} \lambda \mu \\
& +2 \mu^{2}-4 \epsilon_{1} \mu^{2}-4 \epsilon_{2} \mu^{2}+c_{3}\left(-5 e_{2} e_{3}+2 e_{3}^{2}+e_{1}\left(-2 e_{2}+e_{3}\right)+2 \mu-4 \epsilon_{1} \mu-4 \epsilon_{2} \mu\right) \\
& \left.\left.+2 c_{1}\left(e_{2}^{2}-e_{2} e_{3}+\lambda-2 \epsilon_{1} \lambda-2 \epsilon_{2} \lambda+\mu-2 \epsilon_{1} \mu-2 \epsilon_{2} \mu\right)\right)\right) /\left(\left(-2+4 \epsilon_{1}+4 \epsilon_{2}\right) c_{3}^{2}\right. \\
& +c_{3}\left(c _ { 4 } c _ { 3 } \left(c_{4}-2 \epsilon_{1} c_{4}-2 \epsilon_{2} c_{4}-4 e_{1} e_{2}+2 e_{1} e_{3}-10 e_{2} e_{3}+4 e_{3}^{2}+6 \mu\right.\right. \\
& \left.-12 \epsilon_{1} \mu-12 \epsilon_{2} \mu\right)+c_{4}\left(e_{1} e_{2}+5 e_{2}^{2}-2 e_{2} e_{3}+5 \lambda-10 \epsilon_{1} \lambda-10 \epsilon_{2} \lambda+4 \mu-8 \epsilon_{1} \mu-8 \epsilon_{2} \mu\right) \\
& +2\left(2 c_{1} e_{2}^{2}-2 c_{1} e_{2} e_{3}+2 c_{1} \lambda-4 \epsilon_{1} c_{1} \lambda-4 \epsilon_{2} c_{1} \lambda+e_{1}^{2} \lambda\right. \\
& +5 e_{1} e_{3} \lambda+6 e_{3}^{2} \lambda+2 c_{1} \mu-4 \epsilon_{1} c_{1} \mu-4 \epsilon_{2} c_{1} \mu+e_{1}^{2} \mu+3 e_{1} e_{2} \mu+4 e_{2}^{2} \mu \\
& \left.\left.+4 e_{1} e_{3} \mu+4 e_{2} e_{3} \mu+4 e_{3}^{2} \mu+4 \lambda \mu-8 \epsilon_{1} \lambda \mu-8 \epsilon_{2} \lambda \mu+2 \mu^{2}-4 \epsilon_{1} \mu^{2}-4 \epsilon_{2} \mu^{2}\right)\right), \\
& \xi_{7}=\left(4 c_{3} e_{3}\left(\left(-1+2 \epsilon_{1}+2 \epsilon_{2}\right) c_{3}-e_{1} e_{2}-e_{2}^{2}-2 e_{2} e_{3}-\lambda+2 \epsilon_{1} \lambda+2 \epsilon_{2} \lambda\right)\right) /\left(\left(-2+4 \epsilon_{1}+4 \epsilon_{2}\right) c_{3}^{2}\right. \\
& +c_{3}\left(c_{4}-2 \epsilon_{1} c_{4}-2 \epsilon_{2} c_{4}-4 e_{1} e_{2}+2 e_{1} e_{3}-10 e_{2} e_{3}+4 e_{3}^{2}+6 \mu-12 \epsilon_{1} \mu-12 \epsilon_{2} \mu\right) \\
& +c_{4}\left(e_{1} e_{2}+5 e_{2}^{2}-2 e_{2} e_{3}+5 \lambda-10 \epsilon_{1} \lambda-10 \epsilon_{2} \lambda+4 \mu-8 \epsilon_{1} \mu-8 \epsilon_{2} \mu\right) \\
& +2\left(2 c_{1} e_{2}^{2}-2 c_{1} e_{2} e_{3}+2 c_{1} \lambda-4 \epsilon_{1} c_{1} \lambda-4 \epsilon_{2} c_{1} \lambda\right. \\
& +e_{1}^{2} \lambda+5 e_{1} e_{3} \lambda+6 e_{3}^{2}+2 c_{1} \mu-4 \epsilon_{1} c_{1} \mu-4 \epsilon_{2} c_{1} \mu+e_{1}^{2} \mu+3 e_{1} e_{2} \mu+4 e_{2}^{2} \mu \\
& \left.\left.+4 e_{1}^{3} \mu+4 e_{2} e_{3} \mu+4 e_{3}^{2} \mu+4 \lambda \mu-8 \epsilon_{1} \lambda \mu-8 \epsilon_{2} \lambda \mu+2 \mu^{2}-4 \epsilon_{1} \mu^{2}-4 \epsilon_{2} \mu^{2}\right)\right) \text {, } \\
& \xi_{8}=-\frac{e_{2}(3 \lambda+2 \mu)\left(\left(-1+\epsilon_{2}\right) c_{4}+2\left(e_{3}^{2}+\left(-1+\epsilon_{2}\right) \mu\right)\right)}{2 c_{3}(\lambda+\mu)\left(\left(-1+\epsilon_{2}\right) c_{4}-2\left(e_{3}^{2}+\mu-\epsilon_{2} \mu\right)\right)}, \\
& \xi_{9}=-\frac{(3 \lambda+2 \mu)\left(\left(-1+\epsilon_{2}\right) c_{4}+2\left(e_{3}^{2}+\left(-1+\epsilon_{2}\right) \mu\right)\right)}{2(\lambda+\mu)\left(\left(-1+\epsilon_{2}\right) c_{4}-2\left(e_{3}^{2}+\mu-\epsilon_{2} \mu\right)\right)}, \\
& \xi_{10}=\frac{\left(c_{3}+2 \lambda\right)\left(\left(-1+\epsilon_{2}\right) c_{4}+2\left(e_{3}^{2}+\left(-1+\epsilon_{2}\right) \mu\right)\right)}{4(\lambda+\mu)\left(\left(-1+\epsilon_{2}\right) c_{4}-2\left(e_{3}^{2}+\mu-\epsilon_{2} \mu\right)\right)} \\
& \xi_{11}=\frac{c_{3}\left(\left(-1+\epsilon_{2}\right) c_{4}+2\left(e_{3}^{2}-\left(-1+\epsilon_{2}\right)(2 \lambda+\mu)\right)\right)}{4(\lambda+\mu)\left(\left(-1+\epsilon_{2}\right) c_{4}-2\left(e_{3}^{2}+\mu-\epsilon_{2} \mu\right)\right)}, \\
& \xi_{12}=\frac{2 \mu+c_{4}-\epsilon_{2}\left(2 \mu+c_{4}\right)-2 e_{3}^{2}}{2 \epsilon_{2}\left(2 \mu+c_{4}\right)-2\left(c_{4}+2\left(\mu+e_{3}^{2}\right)\right)} \xi_{5}, \\
& \xi_{13}=\frac{e_{2} e_{3}\left(c_{4}+2 \mu\right)(3 \lambda+2 \mu)}{c_{3}(\lambda+\mu)\left(c_{4}-\epsilon_{2} c_{4}+2\left(e_{3}^{2}+\mu-\epsilon_{2} \mu\right)\right)},
\end{aligned}
$$




$$
\begin{aligned}
& \xi_{14}=\frac{e_{3}\left(c_{4}+2 \mu\right)(3 \lambda+2 \mu)}{(\lambda+\mu)\left(c_{4}-\epsilon_{2} c_{4}+2\left(e_{3}^{2}+\mu-\epsilon_{2} \mu\right)\right)}, \\
& \xi_{15}=\frac{e_{3}\left(c_{3}+2 \lambda\right)\left(c_{4}+2 \mu\right)}{2(\lambda+\mu)\left(\left(-1+\epsilon_{2}\right) c_{4}-2\left(e_{3}^{2}+\mu-\epsilon_{2} \mu\right)\right)}, \\
& \xi_{16}=\frac{c_{3} e_{3}\left(c_{4}-2 \lambda\right)}{2(\lambda+\mu)\left(\left(-1+\epsilon_{2}\right) c_{4}-2\left(e_{3}^{2}+\mu-\epsilon_{2} \mu\right)\right)}, \\
& \xi_{17}=-\frac{\left(2 \mu+c_{4}\right) e_{3}}{-c_{4}+\epsilon_{2}\left(2 \mu+c_{4}\right)-2\left(\mu+e_{3}^{2}\right)} \xi_{5}, \\
& \xi_{18}=\frac{1}{4 \lambda+\mu)^{2} c_{3}}\left(\frac{(\lambda+\mu)(3 \lambda+2 \mu)\left(2(\lambda+\mu)+2 c_{3}+c_{4}\right) e_{2}\left(-2 \mu-c_{4}+\epsilon_{2}\left(2 \mu+c_{4}\right)+2 e_{3}^{2}\right)}{-c_{4}+\epsilon_{2}\left(2 \mu+c_{4}\right)-2\left(\mu+e_{3}^{2}\right)}\right. \\
& +\frac{4(\lambda+\mu)(3 \lambda+2 \mu)\left(2 \mu+c_{4}\right) e_{2} e_{3}\left(e_{2}+e_{3}\right)}{c_{4}-\epsilon_{2}\left(2 \mu+c_{4}\right)+2\left(\mu+e_{3}^{2}\right)}-e_{2}\left(-8(\lambda+\mu)^{2} c_{1}-\left(5 \lambda^{2}+11 \lambda \mu+6 \mu^{2}\right) c_{4}\right. \\
& +2\left(3 \lambda^{3}+2 \lambda^{2} \mu-3 \lambda \mu^{2}-2 \mu^{3}+12 \mu^{2} \lambda_{2}+4 \lambda^{2} \lambda_{3}+8 \lambda \mu \lambda_{3}+4 \mu^{2} \lambda_{3}+8 \lambda \mu \lambda_{4}+8 \mu^{2} \lambda_{4}\right. \\
& \left.\left.+4 \lambda^{2} \lambda_{6}+8 \lambda \mu \lambda_{6}+4 \mu^{2} \lambda_{6}-4 \lambda^{2} \lambda_{7}-4 \lambda \mu \lambda_{7}+6 \lambda^{2} \lambda_{8}+4 \lambda \mu \lambda_{8}+4 \mu^{2} \lambda_{8}+3 \lambda^{2} \lambda_{9}\right)\right)-2(\lambda+\mu) \\
& \left.c_{3}\left(2(\lambda+\mu) e_{1}+\lambda e_{2}+4 \lambda e_{3}+4 \mu e_{3}-4 \mu v_{3}+2 \lambda v_{6}-4 \lambda v_{10}-4 \mu v_{10}-2 \lambda v_{14}-\mu v_{14}\right)\right) \text {, } \\
& \xi_{19}=\frac{1}{4 \lambda+\mu)^{2} c_{3}}\left(\frac{(\lambda+\mu)(3 \lambda+2 \mu)\left(2(\lambda+\mu)+2 c_{3}+c_{4}\right)\left(-2 \mu-c_{4}+\epsilon_{2}\left(2 \mu+c_{4}\right)+2 e_{3}^{2}\right)}{-c_{4}+\epsilon_{2}\left(2 \mu+c_{4}\right)-2\left(\mu+e_{3}^{2}\right)}\right. \\
& +\frac{4(\lambda+\mu)(3 \lambda+2 \mu)\left(2 \mu+c_{4}\right) e_{2} e_{3}\left(e_{2}+e_{3}\right)}{c_{4}-\epsilon_{2}\left(2 \mu+c_{4}\right)+2\left(\mu+e_{3}^{2}\right)} \\
& -\frac{1}{e_{2}}\left(e _ { 2 } \left(6 \lambda^{3}+4 \lambda^{2} \mu-6 \lambda \mu^{2}-4 \mu^{3}-8(\lambda+\mu)^{2} c_{1}-\left(5 \lambda^{2} c_{4}+11 \lambda \mu c_{4}-6 \mu^{2} c_{4}\right.\right.\right. \\
& +24 \mu^{2} \lambda_{2}+8 \lambda^{2} \lambda_{3}+16 \lambda \mu \lambda_{3}+8 \mu^{2} \lambda_{3}+16 \lambda \mu \lambda_{4}+16 \mu^{2} \lambda_{4}+8 \mu^{2} \lambda_{4}+8 \lambda^{2} \lambda_{6}+16 \lambda \mu \lambda_{6} \\
& \left.\left.+8 \mu^{2} \lambda_{6}-8 \lambda^{2} \lambda_{7}-8 \lambda \mu \lambda_{7}+12 \lambda^{2} \lambda_{8}+8 \lambda \mu \lambda_{8}+8 \mu^{2} \lambda_{8}+6 \lambda^{2} \lambda_{9}\right)\right)+2(\lambda+\mu) c_{3} \\
& \left.\times\left(2(\lambda+\mu) e_{1}+\lambda e_{2}+4 \lambda e_{3}+4 \mu e_{3}-4 \mu v_{3}+2 \lambda v_{6}-4 \lambda v_{10}-4 \mu v_{10}-2 \lambda v_{14}-\mu v_{14}\right)\right), \\
& \xi_{20}=\frac{1}{2(\lambda+\mu)^{2} c_{3}}\left(\frac{2(\lambda+\mu)(3 \lambda+2 \mu)\left(2 \mu+c_{4}\right) e_{2}^{2} e_{3}}{c_{4}-e_{2}\left(2 \mu+c_{4}\right)+2\left(\mu+e_{3}^{2}\right)}\right. \\
& +\frac{(\lambda+\mu)(3 \lambda+2 \mu)\left(\lambda+c_{3}\right) e_{2}\left(\left(-1+e_{2}\right) c_{4}+2\left(\mu\left(-1+\epsilon_{2}\right)+e_{3}^{2}\right)\right)}{-c_{4}+\epsilon_{2}\left(2 \mu+c_{4}\right)-2\left(\mu+e_{3}^{2}\right)} \\
& -e_{2}\left(3 \lambda^{3}+5 \lambda^{2} \mu+2 \lambda \mu^{2}+\left(\lambda^{2}+3 \lambda \mu+2 \mu^{2}\right) c_{3}+12 \mu^{2} \lambda_{2}+4 \lambda^{2} \lambda_{3}+8 \lambda \mu \lambda_{3}+4 \mu^{2} \lambda_{3}\right. \\
& +8 \lambda \mu \lambda_{4}+8 \mu^{2} \lambda_{4}+4 \lambda^{2} \lambda_{6}+8 \lambda \mu \lambda_{6}+4 \mu^{2} \lambda_{6}-4 \lambda^{2} \lambda_{7}-4 \lambda \mu \lambda_{7}+6 \lambda^{2} \lambda_{8}+4 \lambda \mu \lambda_{8} \\
& \left.\left.+4 \mu^{2} \lambda_{8}+3 \lambda^{2} \lambda_{9}\right)-2(\lambda+\mu) c_{3}\left(-2 \mu v_{3}+\lambda v_{6}-(\lambda+\mu)\left(2 v_{10}+v_{14}\right)\right)\right),
\end{aligned}
$$




$$
\begin{aligned}
& \xi_{21}=\frac{1}{2(\lambda+\mu)^{2}}\left(\frac{2(\lambda+\mu)(3 \lambda+2 \mu)\left(2 \mu+c_{4}\right) e_{2} e_{3}}{c_{4}-\epsilon_{2}\left(2 \mu+c_{4}\right)+2\left(\mu+e_{3}^{2}\right)}\right. \\
& +\frac{(\lambda+\mu)(3 \lambda+2 \mu)\left(\lambda+c_{3}\right)\left(\left(-1+\epsilon_{2}\right) c_{4}+2\left(\mu\left(-1+\epsilon_{2}\right)+e_{3}^{2}\right)\right)}{-c_{4}+\epsilon_{2}\left(2 \mu+c_{4}\right)-2\left(\mu+e_{3}^{2}\right)} \\
& -\frac{1}{e_{2}}\left(e _ { 2 } \left(3 \lambda^{3}+5 \lambda^{2} \mu+2 \lambda \mu^{2}+\left(\lambda^{2}+3 \lambda \mu+2 \mu^{2}\right) c_{3}+12 \mu^{2} \lambda_{2}+4 \lambda^{2} \lambda_{3}+8 \lambda \mu \lambda_{3}+4 \mu^{2} \lambda_{3}\right.\right. \\
& +8 \lambda \mu \lambda_{4}+8 \mu^{2} \lambda_{4}+4 \lambda^{2} \lambda_{6}+8 \lambda \mu \lambda_{6}+4 \mu^{2} \lambda_{6}-4 \lambda^{2} \lambda_{7}-4 \lambda \mu \lambda_{7}+6 \lambda^{2} \lambda_{8}+4 \lambda \mu \lambda_{8}+4 \mu^{2} \lambda_{8} \\
& \left.\left.\left.+3 \lambda^{2} \lambda_{9}\right)+2(\lambda+\mu) c_{3}\left(-2 \mu v_{3}+\lambda v_{6}-2 \lambda v_{10}-2 \mu v_{10}-\lambda v_{14}-\mu v_{14}\right)\right)\right) \text {, } \\
& \xi_{22}=2\left(c_{1}+\lambda / 2+c_{3}+c_{4}+\mu\right)-\left(c_{3}+\lambda+c_{4} / 2+\mu\right)\left(c_{3}+\lambda\right) /(\lambda+\mu), \\
& \xi_{23}=\left(e_{2}+e_{3}\right)\left(c_{3}+\lambda\right) /(\lambda+\mu)-\left(e_{1}+e_{2}+2 e_{3}\right) \text {, } \\
& \xi_{24}=\frac{\left(\lambda+c_{3}\right)\left(2 \mu+c_{4}\right)}{2(\lambda+\mu)}, \\
& \xi_{25}=\frac{\left(\lambda+c_{3}\right) e_{3}}{\lambda+\mu} \\
& \xi_{26}=\frac{2\left(-1+\epsilon_{2}\right)}{-c_{4}+\epsilon_{2}\left(2 \mu+c_{4}\right)-2\left(\mu+e_{3}^{2}\right)}, \\
& \xi_{27}=\frac{2 e_{3}}{c_{4}-\epsilon_{2}\left(2 \mu+c_{4}\right)+2\left(\mu+e_{3}^{2}\right)}, \\
& \xi_{28}=\frac{2 \mu+c_{4}}{c_{4}-\epsilon_{2}\left(2 \mu+c_{4}\right)+2\left(\mu+e_{3}^{2}\right)}, \\
& \xi_{29}=-\left(-\lambda-\mu+2(\lambda+\mu) \epsilon_{1}+2(\lambda+\mu) \epsilon_{2}-e_{2}^{2}\right) /\left(3 \lambda \mu+2 \mu^{2}+2 \lambda c_{1}+2 \mu c_{1}+\lambda c_{3}+2 \mu c_{3}\right. \\
& +2 \lambda c_{4}+2 \mu c_{4}-2 \epsilon_{1}\left(3 \lambda \mu+2 \mu^{2}+2 \lambda c_{1}+2 \mu c_{1}+\lambda c_{3}+2 \mu c_{3}+2 \lambda c_{4}+2 \mu c_{4}\right)-2 \epsilon_{2} \\
& \left(3 \lambda \mu+2 \mu^{2}+2 \lambda c_{1}+2 \mu c_{1}+\lambda c_{3}+2 \mu c_{3}+2 \lambda c_{4}+2 \mu c_{4}\right)+\lambda e_{1}^{2}+\mu e_{1}^{2}+2 \mu e_{1} e_{2}-c_{3} e_{1} e_{2} \\
& \left.+3 \mu e_{2}^{2}+2 c_{1} e_{2}^{2}+c_{3} e_{2}^{2}+2 c_{4} e_{2}^{2}+4 \lambda e_{1} e_{3}+4 \mu e_{1} e_{3}+4 \mu e_{2} e_{3}-2 c_{3} e_{2} e_{3}+4 \lambda e_{3}^{2}+4 \mu e_{3}^{2}\right), \\
& \xi_{30}=-\left((\lambda+\mu) e_{1}+\mu e_{2}+2(\lambda+\mu) e_{3} /\left(3 \lambda \mu+2 \mu^{2}+2 \lambda c_{1}+2 \mu c_{1}+\lambda c_{3}+2 \mu c_{3}\right.\right. \\
& +2 \lambda c_{4}+2 \mu c_{4}-2 \epsilon_{1}\left(3 \lambda \mu+2 \mu^{2}+2 \lambda c_{1}+2 \mu c_{1}+\lambda c_{3}+2 \mu c_{3}+2 \lambda c_{4}+2 \mu c_{4}\right) \\
& -2 \epsilon_{2}\left(3 \lambda \mu+2 \mu^{2}+2 \lambda c_{1}+2 \mu c_{1}+\lambda c_{3}+2 \mu c_{3}+2 \lambda c_{4}+2 \mu c_{4}\right)+\lambda e_{1}^{2}+\mu e_{1}^{2}+2 \mu e_{1} e_{2}-c_{3} e_{1} e_{2} \\
& \left.+3 \mu e_{2}^{2}+2 c_{1} e_{2}^{2}+c_{3} e_{2}^{2}+2 c_{4} e_{2}^{2}+4 \lambda e_{1} e_{3}+4 \mu e_{1} e_{3}+4 \mu e_{2} e_{3}-2 c_{3} e_{2} e_{3}+4 \lambda e_{3}^{2}+4 \mu e_{3}^{2}\right),
\end{aligned}
$$




$$
\begin{aligned}
& \xi_{31}=-\left(3 \lambda \mu+2 \mu^{2}+2(\lambda+\mu) c_{1}+(\lambda+2 \mu) c_{3}+2 \lambda c_{4}+2 \mu c_{4}\right) /\left(3 \lambda \mu+2 \mu^{2}+2 \lambda c_{1}+2 \mu c_{1}+\lambda c_{3}\right. \\
& +2 \mu c_{3}+2 \lambda c_{4}+2 \mu c_{4}-2 \epsilon_{1}\left(3 \lambda \mu+2 \mu^{2}+2(\lambda+\mu) c_{1}+(\lambda+2 \mu) c_{3}+2 \lambda c_{4}+2 \mu c_{4}\right) \\
& -2 \epsilon_{2}\left(3 \lambda \mu+2 \mu^{2}+2(\lambda+\mu) c_{1}+(\lambda+2 \mu) c_{3}+2 \lambda c_{4}+2 \mu c_{4}\right)+\lambda e_{1}^{2}+\mu e_{1}^{2}+2 \mu e_{1} e_{2}-c_{3} e_{1} e_{2} \\
& \left.+3 \mu e_{2}^{2}+2 c_{1} e_{2}^{2}+c_{3} e_{2}^{2}+2 c_{4} e_{2}^{2}+4 \lambda e_{1} e_{3}+4 \mu e_{1} e_{3}+4 \mu e_{2} e_{3}-2 c_{3} e_{2} e_{3}+4 \lambda e_{3}^{2}+4 \mu e_{3}^{2}\right), \\
& \tilde{\mathrm{E}}=-\frac{3 \lambda \mu+2 \mu^{2}+2(\lambda+\mu) c_{1}+(\lambda+2 \mu) c_{3}+2 \lambda c_{4}+2 \mu c_{4}}{2(\lambda+\mu)}, \\
& \xi_{32}=\frac{2\left(\lambda \xi_{19}+c_{3} \xi_{19}+2 \lambda \chi_{55}+2 \mu \chi_{55}\right)}{\lambda\left(3 \lambda+2 \mu+2 c_{1}+4 c_{3}+2 c_{4}\right)}, \\
& \xi_{33}=\frac{2\left(\lambda \xi_{18}+c_{3} \xi_{18}+2 \lambda \chi_{56}+2 \mu \chi_{56}\right)}{\lambda\left(3 \lambda+2 \mu+2 c_{1}+4 c_{3}+2 c_{4}\right)} \text {, } \\
& \xi_{34}=-\frac{2(\lambda+\mu) \chi_{41}}{-\lambda+2 \lambda \epsilon_{1}+2 \lambda \epsilon_{2}-\lambda e_{1}+3 \lambda e_{2}+4 \mu e_{2}-2 \lambda e_{3}}, \\
& \xi_{35}=\frac{2(\lambda+\mu) e_{2}^{2} \chi_{39}}{\lambda c_{3}^{2}-2 \lambda \epsilon_{1} c_{3}^{2}-2 \lambda \epsilon_{2} c_{3}^{2}+\lambda e_{1} e_{2}^{2}-3 \lambda e_{2}^{3}-4 \mu e_{2}^{3}+2 \lambda e_{2}^{2} e_{3}}, \\
& \xi_{36}=\frac{2(\lambda+\mu) c_{3}^{2} \chi_{40}}{\lambda c_{3}^{2} e_{1}-3 \lambda c_{3}^{2} e_{2}-4 \mu c_{3}^{2} e_{2}+\lambda e_{2}^{2}-2 \lambda \epsilon_{1} e_{2}^{2}-2 \lambda \epsilon_{2} e_{2}^{2}+2 \lambda c_{3}^{2} e_{3}}, \\
& \xi_{38}=\left(2 ( \lambda + \mu ) e _ { 2 } \left(e_{1} \xi_{11}+e_{2} \xi_{11}+2 e_{3} \xi_{11}\right.\right. \\
& \left.\left.+e_{1} \xi_{12}+e_{2} \xi_{12}+2 e_{3} \xi_{12}+\xi_{16}-2 \epsilon_{1} \xi_{16}-2 \epsilon_{2} \xi_{16}+\xi_{17}-2 \epsilon_{1} \xi_{17}-2 \epsilon_{2} \xi_{17}+\chi_{42}\right)\right) / \\
& \left(\lambda c_{3}-2 \lambda \epsilon_{1} c_{3}-2 \lambda \epsilon_{2} c_{3}+\lambda e_{1} e_{2}-3 \lambda e_{2}^{2}-4 \mu e_{2}^{2}+2 \lambda e_{2} e_{3}\right), \\
& \xi_{39}=\left(2 ( \lambda + \mu ) e _ { 2 } \left(e_{1} \xi_{10}+e_{2} \xi_{11}+2 e_{3} \xi_{10}\right.\right. \\
& \left.\left.+e_{1} \xi_{12}+e_{2} \xi_{12}+2 e_{3} \xi_{12}+\xi_{15}-2 \epsilon_{1} \xi_{15}-2 \epsilon_{2} \xi_{15}+\xi_{17}-2 \epsilon_{1} \xi_{17}-2 \epsilon_{2} \xi_{17}+\chi_{43}\right)\right) / \\
& \left(\lambda c_{3}-2 \lambda \epsilon_{1} c_{3}-2 \lambda \epsilon_{2} c_{3}+\lambda e_{1} e_{2}-3 \lambda e_{2}^{2}-4 \mu e_{2}^{2}+2 \lambda e_{2} e_{3}\right) \text {. }
\end{aligned}
$$

\section{References}

[1] A.J.C.B Saint-Venant, Mémoire sur la torsion des prismes, Mémoires des Savants étrangers 14 (1856) 233.

[2] A.J.C.B. Saint-Venant, Mémoire sur la flexion des prismes, J. de Mathématiques de Liouville, Ser. II 1 (1956) 89.

[3] D. Iesan, Saint-Venant's problem for inhomogeneous and anisotropic elastic bodies, J. Elasticity 6 (1976) 277-294.

[4] D. Iesan, On Saint-Venant's problem for elastic dielectrics, J. Elasticity 21 (1989) 101.

[5] D. Iesan, Saint-Venant's problem, Springer, New York, 1987.

[6] D. Iesan, L. Nappa, Saint-Venant's problem for microstretch elastic solids, Int. J. Engng.Sci. 32 (1994) 229-236.

[7] F. dell'Isola, L. Rosa, Saint-Venant problem in Linear Piezoelectricity. In: V.V. Varadhan (ed.), Mathematics and Control in Smart Structures, SPIE, vol. 2715, pp. 399-409, 1996.

[8] F. dell'Isola, L. Rosa, Almansi-type boundary conditions for electric potential inducing flexure in linear piezoelectric beams, Cont. Mechs. and Thermodynamics 9 (1997) 115-125.

[9] F. Dávi, Saint-Venant's problem for linear piezoelectric bodies, J. Elasticity 43 (1996) 227-245.

[10] F. dell'Isola, R.C. Batra, Saint-Venant's problem for porous linear elastic materials, J. Elasticity 47 (1997) 73-81.

[11] R.C. Batra, J.S. Yang, Saint-Venant's principle in linear piezoelectricity, J. Elasticity 38 (1995) 209-218.

[12] R.A. Toupin, Saint-Venant's principle, Arch. Rational Mechanics Anal 18 (1965) 83-96.

[13] R.S. Rivlin, The solution of problems in second order elasticity theory, J. Rational Mechs. Analysis 2 (1953) 53-81. 
[14] J.H. Poynting, On pressure perpendicular to the shear-planes in finite pure shears and on the lengthening of loaded wires when twisted, Proc. Roy. Soc. London A82 (1909) 546-549.

[15] A. Signorini, Sulle deformazioni termoelastiche finite, Proc. 3rd Int. Congr. Appl. Mechs. 2 (1930) 80-89.

[16] C.A. Truesdell, W. Noll, S. Flügge (Ed.), The nonlinear field theories of mechanics, Handbuch der Physik, vol. III/ 3, Springer, Berlin, 1965.

[17] A.E. Green, J.E. Adkins, Large Elastic Deformations and Nonlinear Continuum Mechanics, Claredon Press, Oxford, 1960.

[18] A.E. Green, R.T. Shield, Finite extension and torsion of cylinder, Proc. Roy. Soc. London 244 (1951) 47-86.

[19] F. dell'Isola, G.C. Ruta, R.C. Batra, A second-order solution of Saint-Venant's problem for an elastic pretwisted bar using Signorini's perturbation method, J. Elasticity 49 (1998) 113-127.

[20] F. dell'Isola, G.C. Ruta, R.C. Batra, Generalized poynting effects in predeformed-prismatic bars, J. Elasticity 50 (1998) 181-196.

[21] R.C. Batra, F. dell'Isola, S. Vidoli, A second-order solution of Saint-Venant's problem for a piezoelectric circular bar using Signorini's perturbation method, J. Elasticity (in press).

[22] J.S. Yang, R.C. Batra, A second-order theory of piezoelectric materials, J. Acoustic Soc. America 97 (1995) 280288.

[23] A.C. Eringen, G.A. Maugin, Electrodynamics of Continua, Springer, New York, 1989. 